\title{
RISE OF CORRELATIONS OF TRANSFORMATION STRAINS IN RANDOM POLYCRYSTALS*
}

\author{
LEONID BERLYAND ${ }^{\dagger}$, OSCAR BRUNO ${ }^{\ddagger}$, AND ALEXEI NOVIKOV $§$
}

\begin{abstract}
We investigate the statistics of the transformation strains that arise in random martensitic polycrystals as boundary conditions cause its component crystallites to undergo martensitic phase transitions. In our laminated polycrystal model the orientation of the $n$ grains (crystallites) is given by an uncorrelated random array of the orientation angles $\theta_{i}, i=1, \ldots, n$. Under imposed boundary conditions the polycrystal grains may undergo a martensitic transformation. The associated transformation strains $\varepsilon_{i}, i=1, \ldots, n$ depend on the array of orientation angles, and they can be obtained as a solution to a nonlinear optimization problem. While the random variables $\theta_{i}$, $i=1, \ldots, n$ are uncorrelated, the random variables $\varepsilon_{i}, i=1, \ldots, n$ may be correlated. This issue is central in our considerations. We investigate it in following three different scaling limits: (i) Infinitely long grains (laminated polycrystal of height $L=\infty$ ); (ii) Grains of finite but large height $(L \gg 1)$; and (iii) Chain of short grains $\left(L=l_{0} /(2 n), l_{0} \ll 1\right)$. With references to de Finetti's theorem, Riesz' rearrangement inequality, and near neighbor approximations, our analyses establish that under the scaling limits (i), (ii), and (iii) the arrays of transformation strains arising from given boundary conditions exhibit no correlations, long-range correlations, and exponentially decaying short-range correlations, respectively.
\end{abstract}

Key words. polycrystals, misfit, phase transitions, correlations, De Finetti's theorem, Riesz' rearrangement inequality

AMS subject classifications. 35J20, 74N15, 82B44

DOI. $10.1137 / 070679685$

1. Introduction. We investigate the statistics of the transformation strains (misfits) that arise in random martensitic polycrystals as boundary conditions cause its component crystallites to undergo solid-to-solid (martensitic) phase transitions. Martensitic transformations are shape-deforming phase transitions that can be induced in certain alloys as a result of changes in the imposed strains, stresses, or temperatures. These transitions occur when a crystalline solid transforms between its parent phase (austenite) and any of a number of variants of the product phase (martensite). We focus on a setting that, while sufficiently simple to allow for a complete analytical treatment, provides significant insights on the problem: We study laminated polycrystals that consist of sequences of $n$ of grains of rectangular crosssection - of base $1 / n$ and height $L=L(n)$, so that a complete polycrystal is an infinite parallelepiped with rectangular cross-section of base 1 and height $L$. The goal of this work is to provide a rigorous probabilistic theory for the misfit statistics in such polycrystals and, in particular, to provide a rationale for the approximations implicit in

* Received by the editors January 9, 2007; accepted for publication (in revised form) June 24, 2008; published electronically November 19, 2008.

http://www.siam.org/journals/sima/40-4/67968.html

${ }^{\dagger}$ Department of Mathematics, Pennsylvania State University, University Park, PA 16802 (berlyand@math.psu.edu). This author was supported by NSF grants DMS-0204637 and DMS0708324.

${ }^{\ddagger}$ California Institute of Technology, Applied \& Computational Mathematics, 1200 E. California Boulevard, MC 217-50, Pasadena, CA 91125 (bruno@acm.caltech.edu). This author acknowledges support from NSF grant DMS-0408040, AFOSR grant FA9550-05-1-0006, and NRC-JPL award 1263315.

$\S$ Department of Mathematics, Pennsylvania State University, University Park, PA 16802 (anovikov@math.psu.edu). This author was supported by NSF grant DMS-0604600. 


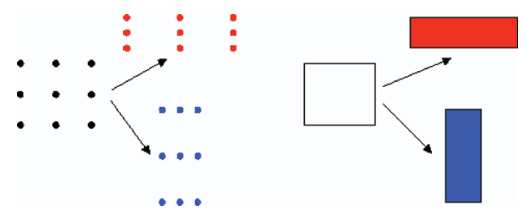

FIG. 1.1. A reference crystallite undergoes stress-free transformations: Atomic view (left) and macroscopic view (right).

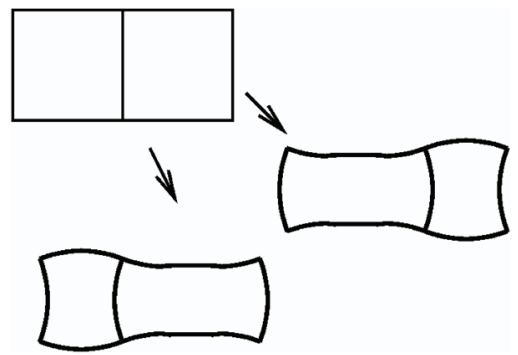

FIG. 1.2. One of two grains undergoes a stress-free transformation.

the numerical algorithms $[6,7,8]$ for polycrytalline phase transitions in two- and three-dimensional space.

The microstructure in a laminated polycrystal is described by a sequence of the orientation angles $\theta_{i}, i=1, \ldots, n ; \theta_{i}$ represents the orientation of the two-dimensional lattice structure in the $i$ th grain. We assume $\theta_{i}$ is a sequence of $n$ independent identically distributed (i.i.d.) random variables. The transformation in the $i$ th grain gives rise to a strain tensor, the transformation strain $\varepsilon_{i}^{\mathrm{T}},(i=1, \ldots, n)$, which is constant, and it takes one of three admissible values: No deformation (the original square lattice remains square), or deformation into one of two rectangular crystalline lattices parallel to the original square lattice. The phase transition in the polycrystal gives rise to a sequence of transformation strains $\varepsilon_{i}^{\mathrm{T}}, i=1, \ldots, n$ obtained by the minimization of the elastic misfit energy among all admissible configurations.

We briefly explain the concept of misfit using a simple example of a polycrystal with two grains. Assume a rectangular single-crystalline grain, considered separately, can undergo a stress-free (two-dimensional version of the) cubic-to-orthorhombic phase deformation into shapes depicted in Figure 1.1. A polycrystal with two square grains can undergo deformations as depicted in Figure 1.2. The elastic energy of the former transformation is zero, because it is stress-free. In contrast, the latter transformation requires some elastic misfit energy that arises because when two crystallites are combined in a polycrystal, their boundaries must remain coherent after the transformation. In general, minimization of misfit energy leads to interactions amongst all of the grains in a polycrystal. Our probabilistic setup allows us to provide a rigorous description of this phenomenology.

The main results of this paper characterize the probability distributions of the random variables $\varepsilon_{i}^{T}$ that arise as minimizers of the overall elastic energy for a given i.i.d. distribution of the angle sequence $\theta_{i}$. Such results are provided in three different cases according to whether the grains are (1) infinitely long $(L=\infty),(2)$ of finite but large height $(L=L \gg 1)$, and (3) short height $\left(L=l_{0} /(2 n), l_{0} \ll 1\right)$. In case (1) our treatment applies to arbitrary i.i.d. probability measures $\rho$ defining the distribution of angles, and in cases (2) and (3), in turn, we restrict consideration to 
i.i.d. distribution of angles with Bernoulli probability measures $\rho$. Our main results can be briefly described as follows:

1. Infinitely long grains. Theorem 5.2. For an arbitrary i.i.d. distribution of angles $\theta_{i}, i=1,2, \ldots, n$, under certain technical assumptions, in the limit $n \rightarrow \infty$, the transformation strains $\varepsilon_{i}^{\mathrm{T}}, i=1,2 \ldots, n$ are also i.i.d. with probability measure $\mu$, where the measure $\mu$ is the minimizer of a certain functional ((5.8) below). In particular, in the case of infinitely long grains there are no correlations between transformation strains of any two grains.

2. Long finite grains. $L \gg 1$. Theorem 6.4. If $\theta_{i}, i=1, \ldots, n$ are Bernoulli random variables (4.3), then in the limit $n \rightarrow \infty, \varepsilon_{i}^{\mathrm{T}}, i=1,2 \ldots, n$ have long-range but no short-range correlations.

3. Short grains. $L=l_{0} /(2 n), l_{0} \ll 1$. Theorems 7.4 and 7.5. If $\theta_{i}, i=1, \ldots, n$ are Bernoulli random variables (4.3), then in the limit $n \rightarrow \infty, \varepsilon_{i}^{\mathrm{T}}, i=$ $1,2 \ldots, n$ have short-range but no long-range correlations.

Results 2 and 3 can be explained as follows. The cornerstone of our study is the maximization of an integral energy functional (see (3.4) below) of the form $\int K_{L}(x-$ t) $f(x) f(t) d x d t$. Its integral kernel $K_{L}(x)$ decays on different length scales for long and short grains. For long grains it decays on the length scale of the composite (on $O(1)$ scale), while for short grains it decays on the length scale of a grain (on $O(1 / n)$ scale). Maximization with respect to this integral kernel leads to long-range and shortrange correlations for long and short grains, respectively. Formally, correlations arise because grains that undergo the stress-free transformation tend to "group together" on the scale of the decay of the integral kernel. We justify this heuristic idea in the case of long grains (see section 6) by applying a randomized version of the Riesz rearrangement inequality. In the case of short grains (see section 7) we show the transforming grains group together-by applying an isoperimetric inequality.

The paper is organized as follows: After describing in section 2 our model of the polycrystal, in section 3 we solve an auxiliary linear elasticity problem, and we obtain an explicit expression for the stored elastic energy for a fixed admissible array of transformation strains. In section 4 we describe our probabilistic model. Our main results are then established in the next three sections, where the nonlinear minimization problem for a random polycrystal is solved. The cases concerning infinitely long grains, finitely long grains, and short grains are studied in sections 5, 6, and 7, respectively.

\section{Formulation.}

Stress-free transformation. A two-dimensional polycrystal is a collection of grains. In our model, each grain is a single crystal (a crystallite) which can undergo a shapedeforming phase transition that results in a transformation strain. An untransformed grain with a horizontal-vertical square lattice (angle $\theta=0$ ) may either elongate in the horizontal direction and remain unchanged in the vertical direction (the upper-right state in Figure 1.1); it may elongate in the vertical direction and remain unchanged in the horizontal direction (the lower right state in Figure 1.1); or, finally, it may not transform at all and thus have its size unchanged (the left state in Figure 1.1). These states correspond to the transformation strains:

$$
\varepsilon_{0}^{1}=\left(\begin{array}{ll}
1 & 0 \\
0 & 0
\end{array}\right), \varepsilon_{0}^{2}=\left(\begin{array}{ll}
0 & 0 \\
0 & 1
\end{array}\right), \varepsilon_{0}^{0}=\left(\begin{array}{ll}
0 & 0 \\
0 & 0
\end{array}\right) .
$$

The first and the second state correspond to a nontrivial transformation. The null strain $\varepsilon_{0}^{0}$ corresponds to absence of transformation. 

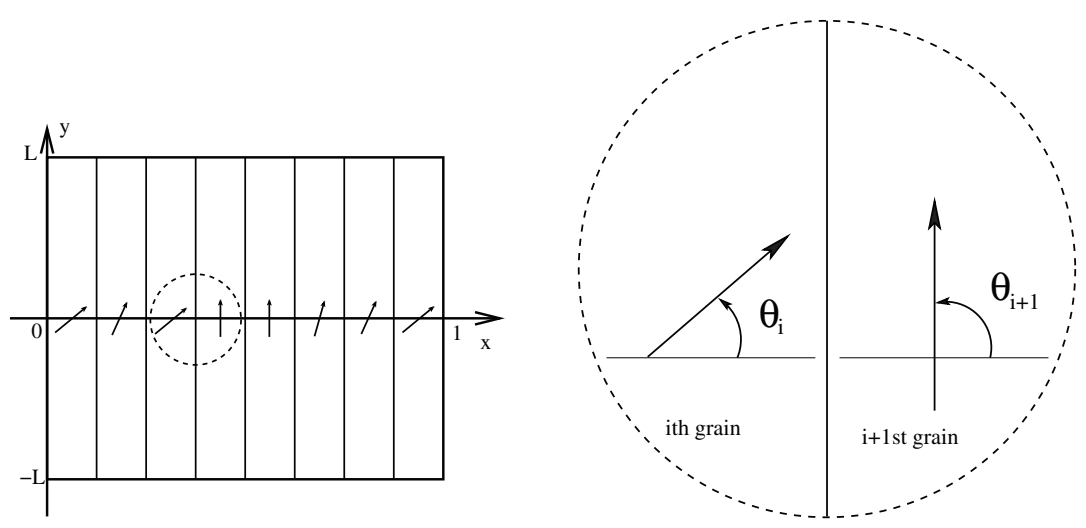

FIG. 2.1. The laminated polycrystal.

Mathematical model of a laminated polycrystal. Grains in a polycrystal have a varying orientation of the crystalline lattices. We consider a rectangular polycrystal $\Pi_{L}=[0,1] \times[-L, L]$ (see Figure 2.1) partitioned into $n$ vertical rectangular layers (the grains) of width $1 / n$ and height $2 L$,

$$
\Pi_{L}=\cup_{i=1}^{n} \Pi_{L}^{i}, \Pi_{L}^{i}=\left[\frac{i-1}{n}, \frac{i}{n}\right] \times[-L, L] .
$$

Each grain $\Pi_{L}^{i}$ is occupied by a crystallite obtained by rotation by the orientation angle

$$
\theta_{i}, 0 \leq \theta_{i} \leq \pi / 2,
$$

of the reference crystallite (see Figure 2.1).

The array of crystallites' orientations is completely determined by the vector of the orientation angles

$$
\boldsymbol{\theta}=\left(\theta_{1}, \theta_{2}, \ldots, \theta_{n}\right)
$$

Using the matrix of rotation by an angle $\theta$

$$
R=R(\theta)=\left(\begin{array}{cc}
\cos \theta & \sin \theta \\
-\sin \theta & \cos \theta
\end{array}\right)
$$

we see that the stress-free transformation strain for the grain $\Pi_{L}^{i}$ must lie in the set

$$
\mathcal{S}_{\theta_{i}}=\left\{\varepsilon^{1}\left(\theta_{i}\right), \varepsilon^{2}\left(\theta_{i}\right), \varepsilon^{0}\left(\theta_{i}\right)\right\}, 0 \leq \theta_{i} \leq \pi / 2,
$$

where

$$
\begin{aligned}
& \varepsilon^{1}(\theta)=R \varepsilon^{1}(0) R^{t}=\left(\begin{array}{cc}
\cos ^{2}(\theta) & \sin (\theta) \cos (\theta) \\
\sin (\theta) \cos (\theta) & \sin ^{2}(\theta)
\end{array}\right) \\
& \varepsilon^{2}(\theta)=R \varepsilon^{2}(0) R^{t}=\left(\begin{array}{cc}
\sin ^{2}(\theta) & -\sin (\theta) \cos (\theta) \\
-\sin (\theta) \cos (\theta) & \cos ^{2}(\theta)
\end{array}\right), \\
& \varepsilon^{0}(\theta)=R \varepsilon^{0}(0) R^{t}=\left(\begin{array}{ll}
0 & 0 \\
0 & 0
\end{array}\right) .
\end{aligned}
$$

Copyright (c) by SIAM. Unauthorized reproduction of this article is prohibited. 
The superscript $t$ stands for the matrix transpose. The set of all sequences of strains that are admissible for some sequence of angles is denoted by $\tilde{\Omega}_{n}$ :

$$
\tilde{\Omega}_{n}=\left\{\varepsilon^{\mathrm{T}} \mid \varepsilon^{\mathrm{T}}=\left(\varepsilon_{1}^{\mathrm{T}}, \varepsilon_{2}^{\mathrm{T}}, \varepsilon_{3}^{\mathrm{T}}, \ldots, \varepsilon_{n}^{\mathrm{T}}\right), \boldsymbol{\varepsilon}_{i}^{\mathrm{T}} \in \mathcal{S}_{\theta_{i}} \text { for some } \theta_{i} \in[0, \pi / 2]\right\} .
$$

The set of all sequences of strains that are admissible for a given sequence $\boldsymbol{\theta}$ will be denoted by

$$
\tilde{\Omega}_{n}(\boldsymbol{\theta})=\left\{\varepsilon^{\mathrm{T}} \mid \varepsilon^{\mathrm{T}} \in \tilde{\Omega}_{n} \text { such that } \varepsilon_{i}^{\mathrm{T}} \in \mathcal{S}_{\theta_{i}}\right\} .
$$

Linear elasticity equations for given transformation strains. For a given sequence of the orientation angles $\boldsymbol{\theta}=\left\{\theta_{i}, i=1, \ldots, n\right\}$ there are up to $3^{n}$ corresponding sequences $\varepsilon^{\mathrm{T}}=\left\{\boldsymbol{\varepsilon}_{i}^{\mathrm{T}}, i=1, \ldots, n\right\}$ in the class $\tilde{\Omega}_{n}(\boldsymbol{\theta})$ defined in (2.6). Here we introduce the relevant elasticity PDEs on the domain $\Pi_{L}$ for a given such $\varepsilon^{\mathrm{T}}$. We assume that each grain can be described by isotropic elasticity equations with elastic moduli given by

$$
c_{i j k l}=\lambda \delta_{i j} \delta_{k l}+\mathrm{G}\left(\delta_{i k} \delta_{j l}+\delta_{i l} \delta_{j k}\right),
$$

where $\lambda$ and $\mathrm{G}$ are the Lamé constants [19].

As an applied displacement is imposed, our polycrystal may acquire microscopic strains $\varepsilon$ which contain combined contributions of elastic and stress-free transformations (see [9]):

$$
\varepsilon=\varepsilon^{\text {elastic }}+\varepsilon^{\mathrm{T}} .
$$

Then Hooke's law $\sigma_{i j}=c_{i j k l} \varepsilon_{k l}^{\text {elastic }}$ yields the stress-strain relation of linear elasticity under a given transformation strain $\varepsilon^{\mathrm{T}}$

$$
\sigma_{i j}=c_{i j k l}\left(\varepsilon_{k l}-\varepsilon_{k l}^{\mathrm{T}}\right) .
$$

Here the strain tensor $\varepsilon_{k l}$ is determined by the displacement vector $\boldsymbol{u}=\left(u_{1}(x, y)\right.$, $\left.u_{2}(x, y)\right)$ :

$$
\varepsilon_{i j}=\frac{1}{2}\left(\partial_{i} u_{j}+\partial_{j} u_{i}\right) \text { where } \partial_{1}=\frac{\partial}{\partial x}, \partial_{2}=\frac{\partial}{\partial y} .
$$

The stress tensor satisfies the elasticity equations

$$
\partial_{j} \sigma_{i j}=0 \text { for } i=1,2 .
$$

The above equation is to be understood in the distributional sense, and thus the traction must be continuous across the interfaces between grains:

$$
\left[\sigma_{i 1}\right](x, y)=0, \text { for } i=1,2 \text { and } x=m / n, m=1,2, \ldots, n-1 .
$$

For such a given admissible configuration $\varepsilon_{i}^{\mathrm{T}}$, we assume a given imposed displacement that is chosen in the direction transversal to the laminates: ${ }^{1}$

$$
u_{1}(0, y)=0, u_{1}(1, y)=U, u_{2}(0,0)=0,
$$

together with the zero-traction boundary conditions

$$
\sigma_{12}(0, y)=\sigma_{12}(1, y)=0, \sigma_{i 2}(x, \pm L)=0 .
$$

\footnotetext{
${ }^{1}$ Other boundary conditions (e.g., shears) could be treated similarly.
} 
It is easy to check that, for a fixed admissible configuration $\varepsilon^{\mathrm{T}},(2.11),(2.13)$, and (2.14) are the Euler-Lagrange equations for the minimizer of the elastic energy

$$
W\left(U, \boldsymbol{\varepsilon}^{\mathrm{T}}\right)=\frac{1}{E_{c}} \min _{\boldsymbol{u}} \frac{1}{2 L} \int_{\Pi_{L}}\left(\varepsilon_{i j}-\varepsilon_{i j}^{\mathrm{T}}\right) c_{i j k l}\left(\varepsilon_{k l}-\varepsilon_{k l}^{\mathrm{T}}\right) d x d y, \quad \boldsymbol{u} \text { subject to (2.13), }
$$

where

$$
E_{c}=\frac{4 \mathrm{G}(\lambda+\mathrm{G})}{\lambda+2 \mathrm{G}}
$$

is the two-dimensional Young modulus. Thus it can be verified that the boundary value problem (2.11), (2.13), and (2.14) admits a unique solution.

Overall polycrystalline energy. As a displacement (2.13) is imposed on the polycrystal, each grain may undergo a stress-free transformation into one of the three possible stress-free states. The overall energy $\mathcal{W}_{n}(U, \boldsymbol{\theta})$ in the polycrystal is determined by global minimization of the misfit energy $W\left(U, \varepsilon^{\mathrm{T}}\right)$ of the polycrystal over all admissible configurations [9]

$$
\mathcal{W}_{n}(U, \boldsymbol{\theta})=\min _{\left.\boldsymbol{\varepsilon}^{\mathrm{T}} \in \tilde{\Omega}_{n}\right|_{\theta}} W\left(U, \varepsilon^{\mathrm{T}}\right) .
$$

The (possible nonunique) array(s) of transformations strains that arise in the polycrystal is (are) the minimizer(s) in (2.17).

Simplifying assumptions of our model. The idea that the energy minimization in composites and polycrystals can explain correlations has been long pursued in material science (see, e.g., $[16,22]$ ). In this paper we use a quadratic form of the polycrystal's energy proposed in [9] and further developed analytically in [20,6] and numerically in $[6,7,8]$.

The probabilistic model introduced in this work captures many of the essential features of the general physical phenomenon of misfit and, at the same time, is amenable to rigorous analytical treatment.

Clearly, however, our model is too simple to reflect the rich phenomena that occur in actual three-dimensional polycrystals. For example, we consider isotropic elasticity, whereas typically, the crystalline lattice of each of the martensite variants has less symmetry than that of the austenite. Further, for sufficiently large grains, the lattices associated with the various martensite variants could be combined, giving rise to twins and/or higher-rank laminates of two or more different variants of martensite within each grain $[21,4,15,5,17]$ —an effect that our model does not allow. We also note that, in general, a stress-free transformation is a time-dependent process that involves energy dissipation. Our study assumes that the final state of a polycrystal is determined by minimizers of a time-independent, dissipation-free misfit energy (see, e.g., $[9,6,8]$ and references therein). Importantly, however, we do not assume that the grains in the polycrystal transform without elastic stresses (self-accommodation); see, e.g., $[3,2]$ and references therein.

Although not explicitly considered in this work, related phenomena, including electrical and magnetic polarizations in electro- and magneto-rheological materials and the combined elastic and magnetic-electric misfits arising from magnetostriction and electrostriction in composite materials, could be treated by similar methods.

3. Elasticity kernel. In this section we give a representation for the elastic energy $W\left(U, \varepsilon^{\mathrm{T}}\right)$ in terms of a certain integral kernel $K_{L}((x-t))$, and we then present 
asymptotics of this kernel under two regimes that are relevant in our studies of the statistics of transformation strains in sections 5, 6, and 7. Denote spatial averages as

$$
\langle g\rangle=\frac{1}{\left|\Pi_{L}\right|} \int_{\Pi_{L}} g(x, y) d x d y=\frac{1}{2 L} \int_{\Pi_{L}} g(x, y) d x d y .
$$

It turns out that the most convenient mathematical formulation of the elastic energy is in terms of

$$
s(x)=\varepsilon_{22}^{\mathrm{T}}(x),
$$

and the volume fraction of grains that undergo a phase transition ${ }^{2}$

$$
f=\langle I\rangle, I=\varepsilon_{11}^{\mathrm{T}}+\varepsilon_{22}^{\mathrm{T}} .
$$

Then the elastic energy

$$
W\left(U, \varepsilon^{\mathrm{T}}\right)=\left\langle(U-f+s)^{2}\right\rangle-\int_{-1}^{1} \int_{-1}^{1}(s(x)-\langle s\rangle) K_{L}((x-t))(s(t)-\langle s\rangle) d x d t
$$

where $K_{L}$ is an even, 2-periodic integral kernel whose cosine Fourier coefficients

$$
\hat{K}_{L}(m)=\int_{-1}^{1} K_{L}(x) \cos (\pi m x) d x
$$

are explicitly given in Appendix A.1 by formulas (A.5) and (A.6).

The idea of the proof of (3.4) is to decompose the solution $\boldsymbol{u}=\left(u_{1}, u_{2}\right)$ of the boundary value problem (2.11), (2.13), and (2.14) in the form $\boldsymbol{u}=\tilde{\boldsymbol{u}}+\overline{\boldsymbol{u}}$, where $\tilde{\boldsymbol{u}}$ solves the elasticity equations (2.15) for infinitely long grains $(L=\infty)$ and the remainder $\overline{\boldsymbol{u}}$ is the correction for finite $L$. It turns out that $\tilde{\boldsymbol{u}}$ is a piecewise linear function of the form

$$
\left\{\begin{array}{l}
\tilde{u}_{1}^{i}=a_{i} x+f_{i}, \\
\tilde{u}_{2}^{i}=c_{i} x+d y+g_{i},
\end{array}\right.
$$

and $\overline{\boldsymbol{u}}$ satisfies the boundary value problem

$$
\left\{\begin{array}{l}
\partial_{j} \bar{\sigma}_{i j}=0 \text { for } i=1,2 \\
\bar{u}_{1}(0, y)=\bar{u}_{1}(1, y)=0, \quad \partial_{1} \bar{u}_{2}(0, y)=\partial_{1} \bar{u}_{2}(1, y)=0 \\
\bar{\sigma}_{12}(x, \pm L)=0, \quad \bar{\sigma}_{22}(x, \pm L)=E_{c}\left(\varepsilon_{22}^{\mathrm{T}}-\left\langle\varepsilon_{22}^{\mathrm{T}}\right\rangle\right)
\end{array}\right.
$$

where

$$
\left\{\begin{array}{l}
\bar{\sigma}_{i j}=c_{i j k l} \bar{\varepsilon}_{k l}, \\
\bar{\varepsilon}_{11}=\partial_{1} \bar{u}_{1}, \quad \bar{\varepsilon}_{12}=\bar{\varepsilon}_{21}=\left(\partial_{2} \bar{u}_{1}+\partial_{1} \bar{u}_{2}\right) / 2, \quad \bar{\varepsilon}_{22}=\partial_{2} \bar{u}_{2} .
\end{array}\right.
$$

Both functions can be computed explicitly: $\tilde{\boldsymbol{u}}$ is obtained from direct computations and $\overline{\boldsymbol{u}}$ is found by the Airy function method. A detailed proof is provided in Appendix A.1. The asymptotics of the convolution kernel in two important limiting

\footnotetext{
${ }^{2}$ Since the transformation strains defined in (2.4) satisfy $\varepsilon_{11}^{1}+\varepsilon_{22}^{1}=1, \varepsilon_{11}^{2}+\varepsilon_{22}^{2}=1$, and $\varepsilon_{11}^{0}+\varepsilon_{22}^{0}=0$, it follows that the quantity $f$ equals the volume fraction of grains that undergo a phase transition.
}

Copyright $@$ by SIAM. Unauthorized reproduction of this article is prohibited. 
cases, in turn, are summarized in the following two lemmas. The proofs of the lemmas are provided in Appendices A.2 and A.3, respectively.

Lemma 3.1 (kernel asymptotics as $L \rightarrow \infty$ ). $K_{L}(x)$ can be represented in the form

$$
K_{L}(x)=\frac{B}{L} K_{\infty}(x)+O(\exp (-L)), \text { as } L \rightarrow \infty,
$$

where

$$
K_{\infty}=-\ln |\sin (\pi x / 2)|,
$$

and

$$
B=\frac{5 \lambda+9 G}{4 \pi(\lambda+2 G)}>0
$$

We now consider polycrystals for which the height is commensurate with the grain widths $L=l_{0} /(2 n)$. The parameter $l_{0}$ is the height-to-width ratio. In particular, when $L=1 /(2 n)$, such polycrystals can be viewed as chains of square grains.

Lemma 3.2 (kernel asymptotics as $L \rightarrow 0$ ). Suppose $L=l_{0} /(2 n)$, where $l_{0}>0$, and $n \rightarrow \infty$. Then for each fixed height-to-width ratio $l_{0}$ there exists a positive-definite function $\mathcal{K}_{l_{0}}(x), x \in \mathbb{R}$ independent of $n$, such that

$$
\left\|K_{L}(x)-n \mathcal{K}_{l_{0}}(n x)\right\|_{L_{\infty}([-1,1])} \rightarrow 0 \text {, as } n \rightarrow \infty,
$$

where $\mathcal{K}_{l_{0}}(x)$ is even: $\mathcal{K}_{l_{0}}(-x)=\mathcal{K}_{l_{0}}(x)$, and

$$
\left|\mathcal{K}_{l_{0}}(x)\right| \leq \frac{c}{l_{0}} \exp \left(-|x| / l_{0}\right) .
$$

The constant $c$ in (3.10) does not depend on $n$ and $l_{0}$.

We will find it useful, especially for chains of rectangular grains, to identify sequences (vectors) $\left(f_{1}, \ldots f_{n}\right)$ (of real number, matrices, etc.) with the corresponding piecewise constant (real valued, matrix valued, etc.) functions defined in the interval $[0,1]$ that take the values $f(x)=f_{k}$ for $x \in \Pi_{L}^{k}, k=1, \ldots, n$. For example, the argument $\varepsilon^{\mathrm{T}}$ of $W$ in (2.15), which is a matrix-valued function defined in the interval $[0,1]$, will often be replaced by a sequence of $n$ matrices $\left(\varepsilon_{1}^{\mathrm{T}}, \varepsilon_{2}^{\mathrm{T}}, \varepsilon_{3}^{\mathrm{T}}, \ldots, \varepsilon_{n}^{\mathrm{T}}\right)$. As another example, note that the dependence on $n$ of the quantity on the left-hand side of (2.17) arises merely from the fact that $\varepsilon^{\mathrm{T}}$ on the right-hand side of that formula is a piecewise constant function determined by a sequence of $n$ matrices. For a function $f$ defined by a sequence $\left(f_{1}, \ldots f_{n}\right)$ the spatial average $(3.1)$ is

$$
\langle f\rangle=\frac{1}{n} \sum_{i=1}^{n} f_{i} .
$$

Further, on the space of the piecewise constant functions the integral representation of the misfit energy (3.4) can be viewed as an algebraic nonnegative definite quadratic form:

$$
W_{n}\left(U, \varepsilon^{\mathrm{T}}\right)=\left\langle(U-f+s)^{2}\right\rangle-\langle(s-\langle s\rangle) M(n, L)(s-\langle s\rangle)\rangle,
$$

where $M=M(n, L)$ is a $n \times n$ symmetric Toeplitz matrix with entries $M_{i j}(n, L)=$ $\lambda_{i-j}(n, L)$ defined by

$$
(s-\langle s\rangle) M(s-\langle s\rangle)=\int_{-1}^{1} \int_{-1}^{1}(s(x)-\langle s\rangle) K_{L}(x-t)(s(t)-\langle s\rangle) d x d t
$$

for piecewise constant functions $s=\left(s_{1}, \ldots, s_{n}\right)$. 
As an immediate consequence of Lemma 3.2 we can estimate the decay of the coefficients $\lambda_{i-j}(n, L)$ for a chain of rectangular grains $\left(L=l_{0} /(2 n)\right)$ : As $n \rightarrow \infty$, $\lambda_{k}(n, L) \rightarrow \lambda_{k}\left(l_{0}\right)$ where

$$
\left|\lambda_{k}\left(l_{0}\right)\right| \leq \frac{c}{n} \exp \left(-|k| / l_{0}\right)
$$

By (3.13) for a chain of rectangular grains $\lambda_{i-j}(n, L)$ can be accurately approximated by a (truncated) Toeplitz matrix by setting $\lambda_{k}(n, L) \equiv 0$, for $|k|>k_{0}$, and the misfit energy is approximately

$$
W_{n}^{k_{0}}\left(U, \varepsilon^{\mathrm{T}}\right)=\left\langle(U-f+s)^{2}\right\rangle-\sum_{k=-k_{0}}^{k_{0}} \sum_{i=1}^{n} \lambda_{k}(n)\left(s_{i}-\langle s\rangle\right)\left(s_{i+k}-\langle s\rangle\right),
$$

where we define $s_{i+k}$ for $i+k>n$ by periodicity

$$
s_{i+k}=\left\{\begin{array}{l}
s_{i+k}, \text { if } i+k \leq n, \\
s_{i+k-n}, \text { if } i+k>n .
\end{array}\right.
$$

The approximation (3.14) provides a justification, in a one-dimensional context, of numerical schemes which are used in practical evaluation of the misfit energy $[9,6,7,8]$. The approximation (3.14) takes into account only interaction with the nearest neighbors. Hence we call (3.14) $k_{0}$-nearest neighbors energy. The next proposition shows that for any finite value of $n$ and finite $k_{0}$, this $k_{0}$-nearest neighbors approximation has an exponential in $k_{0}$ error. Therefore the computational complexity of finding the misfit energy can be significantly reduced if (3.11) is replaced by $(3.14)$. In $[6,7,8]$ this truncation was implemented for general two- and three-dimensional polycrystals, and the convergence was verified numerically. The following proposition justifies this convergence analytically in the case of chains of rectangular grains, and provides an explicit exponential error estimate.

Proposition 3.3. For a given $U$ and a given vector of orientation angles $\boldsymbol{\theta}$, suppose $\boldsymbol{\varepsilon}^{T}\left(k_{0}\right) \in \tilde{\Omega}_{n}(\boldsymbol{\theta})$ is a minimizer of the $k_{0}$-nearest neighbors energy $W_{n}^{k_{0}}\left(U, \varepsilon^{T}\right)$ given by (3.14). Then there is a universal constant $c$, independent of $n$, such that $W_{n}(U, \boldsymbol{\theta})$, the minimum of the misfit energy (3.11), satisfies

$$
\left|W_{n}(U, \boldsymbol{\theta})-W_{n}^{k_{0}}\left(U, \varepsilon^{T}\left(k_{0}\right)\right)\right| \leq c \exp \left(-k_{0}\right) .
$$

A proof the theorem is given in Appendix A.4. Finally, applying Proposition 3.3 and Lemma 3.2, the misfit energy (3.4) of a chain of short grains becomes

$$
\begin{array}{r}
W_{n}\left(U, \varepsilon^{\mathrm{T}}\right)=\left\langle(U-f+s)^{2}\right\rangle-\lambda_{0}\left\langle(s-\langle s\rangle)^{2}\right\rangle-\frac{B e^{-1 / l_{0}}}{n} \sum_{i=1}^{n} \\
\left(s_{i}-\langle s\rangle\right)\left(s_{i+1}-\langle s\rangle\right)+O\left(e^{-2 / l_{0}}\right), \text { as } l_{0} \rightarrow 0,
\end{array}
$$

where $\lambda_{0}>0, B>0 .{ }^{3}$ Thus, when $l_{0} \ll 1$, the misfit energy is approximated by the nearest neighbor energy.

\footnotetext{
${ }^{3}$ From numerical computations $\lambda_{k}, k \geq 2$ are negligible even for large $l_{0}=1: \sum_{k=2}^{\infty}\left|\lambda_{k}\right| \leq .1 \lambda_{1}$, $\lambda_{1} \leq .17 \lambda_{0}$.
} 
4. A probabilistic model. Our probabilistic model is set to describe energy minimizers within a random setting. In detail, we consider orientation angles $\theta_{i}$, $i=1,2, \ldots$ as a sequence of random variables ${ }^{4}$

$$
\theta_{i}: \Omega \rightarrow \Omega^{\theta}=[0, \pi / 4]
$$

on a common probability space $(\Omega, \mathcal{F}, P)$. We denote by $\rho_{n}$ the usual induced probability measure of a sequence of the first $n$ orientation angles on $\left(\Omega_{n}^{\theta}, \sigma_{n}^{\theta}\right)$, where $\Omega_{n}^{\theta}=\left(\Omega^{\theta}\right)^{n}=\Omega^{\theta} \times \Omega^{\theta} \times \cdots \times \Omega^{\theta}$, and $\sigma_{n}^{\theta}$ is the Borel $\sigma$-algebra on $\Omega_{n}^{\theta}$.

We may define and work with the probability space $\left(\tilde{\Omega}_{n}, \tilde{\sigma}_{n}, \tilde{\mu}_{n}\right)$ of arrays of transformation strains $\left(\varepsilon_{i}^{\mathrm{T}}\right)_{i=1}^{n}$, where we recall that $\tilde{\Omega}_{n}$ is the set of all sequences of strains that are admissible for some sequence of orientation angles (see (2.5)).

We will, however, work with a different, but equivalent probability space. Recall that the misfit energy (3.4) depends only on a sequence of pairs $\left\{\left(s_{i}, I_{i}\right)\right\}, i=1, \ldots, n$, where

$$
I_{i}=\varepsilon_{11, i}^{\mathrm{T}}+\varepsilon_{22, i}^{\mathrm{T}} \in\{0,1\}, \text { and } s_{i}=\varepsilon_{22, i}^{\mathrm{T}} \in[0,1] .
$$

Therefore, we will study the probability space

$$
\left(\Omega_{n}^{\mathrm{T}}, \sigma_{n}^{\mathrm{T}}, \mu_{n}\right), \Omega_{n}^{\mathrm{T}}=\left(\Omega^{\mathrm{T}}\right)^{n}=\Omega^{\mathrm{T}} \times \Omega^{\mathrm{T}} \times \cdots \times \Omega^{\mathrm{T}},\left(s_{i}, I_{i}\right) \in \Omega^{\mathrm{T}}=[0,1] \times\{0,1\},
$$

where $\sigma_{n}^{\mathrm{T}}$ is the Borel $\sigma$-algebra on $\Omega_{n}^{\mathrm{T}}$ and $\mu_{n}$ is a probability measure, which we will define next.

Probability measure in the space of transformation strains. Suppose the applied deformation $U$ is given. For a fixed sequence of orientation angles $\boldsymbol{\theta}$, there are up to $3^{n}$ different admissible arrays of transformation strains $\left(\varepsilon_{i}^{\mathrm{T}}\right)_{i=1}^{n}, \mathrm{~T}=0,1,2$; see $(2.6)$ with (2.3) and (2.4). They correspond to $3^{n}$ different arrays of pairs $\left\{\left(s_{i}, I_{i}\right)\right\}, i=$ $1, \ldots, n$ using the rule that the matrices $\varepsilon^{1}\left(\theta_{i}\right), \varepsilon^{2}\left(\theta_{i}\right)$, and $\varepsilon^{0}\left(\theta_{i}\right)$ in (2.4) correspond to the pairs $\left(s_{i}, I_{i}\right)=\left(\sin ^{2} \theta_{i}, 1\right),\left(s_{i}, I_{i}\right)=\left(\cos ^{2} \theta_{i}, 1\right)$, and $\left(s_{i}, I_{i}\right)=(0,0)$, respectively. Some of these arrays $\left\{\left(s_{i}, I_{i}\right)\right\}, i=1, \ldots, n$, say a number $k$ of them, minimize the misfit energy (3.4) amongst all admissible arrays. We assume, as it may indeed be natural from a physics perspective, that each of these energy minimizing arrays occurs with equal probability. In other words, we will define the probability measure $\mu_{n}$ in such a way that the conditional probability measure $\mu_{n}\left(\left(s_{i}, I_{i}\right)_{i=1}^{n} \mid \boldsymbol{\theta}\right)$ satisfies

$$
\mu_{n}\left(\left(s_{i}, I_{i}\right)_{i=1}^{n} \mid \boldsymbol{\theta}\right)=\left\{\begin{array}{l}
1 / k, \text { if } \boldsymbol{\varepsilon}^{\mathrm{T}} \text { is a minimizer } \\
0, \text { if } \boldsymbol{\varepsilon}^{\mathrm{T}} \text { is not a minimizer }
\end{array}\right.
$$

where $k$ is the number of minimizers of the misfit energy (3.4) for the fixed $\boldsymbol{\theta}$. The probability measure $\mu_{n}$ on the sequences $\left(s_{i}, I_{i}\right)_{i=1}^{n}$ is thus defined by

$$
\mu_{n}(A)=\int_{\Omega_{n}^{\theta}} \mu_{n}(A \mid \boldsymbol{\theta}) \mathrm{d} \rho_{n}(\boldsymbol{\theta})
$$

for any Borel set $A \in \sigma_{n}^{\mathrm{T}}$.

\footnotetext{
${ }^{4}$ In principle $\theta \in[0, \pi / 2]$. It follows from (2.4) that we are concerned only with $\sin ^{2} \theta$ and $\cos ^{2} \theta$. Since $\sin (\pi / 2-\theta)=\cos \theta$ and $\cos (\pi / 2-\theta)=\sin \theta$, we may and do assume that $\theta \in[0, \pi / 4]$. The orientation of a square crystalline lattice can be described uniquely by a value $\theta \in[0, \pi / 4]$.
} 
The measure $\mu_{n}$ describes statistics of the transformation strains; the main objective of this paper is to describe it in detail for $n \gg 1$ and for various polycrystal configurations.

Distribution of angles. In the remainder of this paper we will assume that the angles $\theta_{i}$ are independent and identically distributed with the induced probability measure (distribution) $\rho$ on $\Omega^{\theta}=[0, \pi / 4]$-although other types of angle distributions could be considered within the present context. In other words, the probability measure $\rho_{n}$ on the sequences of the orientation angles will be taken to be a product measure of the form

$$
\rho_{n}=\Pi_{i=1}^{n} \rho .
$$

In particular, to illustrate our theory we will consider two specific probability distributions $\rho$ : (1) the uniform distribution, in which $\rho$ is proportional to the Lebesgue measure, and (2) the Bernoulli trials model for which $\rho$ is concentrated in two $\theta$ values

$$
\theta_{i}= \begin{cases}\alpha, & \text { with probability } q, \\ \beta, & \text { with probability } 1-q,\end{cases}
$$

$0 \leq \beta \leq \alpha \leq \pi / 4$

5. Statistics of asymptotic energy minimizers 1: Infinitely long grains $(L=\infty)$.

5.1. The main theorem. Suppose the grains are infinitely long $(L=\infty)$. Then, by Lemma 3.1 the misfit energy for a given admissible sequence of transformation strains on the array of $n$ grains is given by

$$
W_{n}\left(U, \varepsilon^{\mathrm{T}}\right)=\left\langle\left(U-f+\varepsilon_{22}^{\mathrm{T}}\right)^{2}\right\rangle=\left\langle(U-f+s)^{2}\right\rangle .
$$

The sequence of measures (4.2) contains convergent subsequences [18]; each such limit $\mu_{\mathrm{lim}}$ is a measure on the set of infinite sequences of transformation strains; the limits along various subsequences may, in principle, not all coincide. In fact, in all cases we consider, however, all such limits do coincide, and the full sequences (4.2) are convergent. For the sake of simplicity, in the subsequent analysis we assume this is the case and we denote $\mu_{\text {lim }}=\lim _{n \rightarrow \infty} \mu_{n}$.

As we shall show the limits $\mu_{\text {lim }}$ are convex combinations of product measures. This is a consequence of the de Finetti's representation theorem (see [13] for a general version of this theorem). In order to motivate the advantages of this observation in our context, we first consider one such limit $\mu_{\text {lim }}$ and we assume (this may or may not be true!) that (1) for each finite $n$ the minimizers are unique, and (2) the measure $\mu_{\text {lim }}$ is given by a product of the form

$$
\mu_{\lim }=\tilde{\mu}:=\Pi_{i=1}^{\infty} \mu,
$$

for a certain measure $\mu$ so that, according to $\mu_{\mathrm{lim}}$, the random variables $\left\{\left(s_{i}, I_{i}\right)\right\}_{i=1}^{\infty}$ are i.i.d.

Since, as we have seen above, for each $i$ we must necessarily have $s_{i}=\sin ^{2}\left(\theta_{i}\right)$ or $s_{i}=\cos ^{2}\left(\theta_{i}\right)$ whenever $I_{i}=1$, under the uniqueness assumption (1) above the measure $\mu$ in (5.2) must satisfy

$$
\left\{\begin{array}{l}
\mu\left(s=\sin ^{2}(\theta) \mid \theta, I=1\right)+\mu\left(s(\theta)=\cos ^{2}(\theta) \mid \theta, I=1\right)=1 \\
\mu\left(s=\sin ^{2}(\theta) \mid \theta, I=1\right)=0 \quad \text { or } \quad 1 \quad \text { and } \quad \mu\left(s(\theta)=\cos ^{2}(\theta) \mid \theta, I=1\right)=0 \quad \text { or } 1 .
\end{array}\right.
$$


Hence, we can define a function $\kappa(\theta), \kappa(\theta)=0$, or $\kappa(\theta)=1$ such that

$$
\kappa(\theta)= \begin{cases}1, & \text { if } \mu\left(s=\sin ^{2}(\theta) \mid \theta, I=1\right)=1 \\ 0, & \text { if } \mu\left(s=\cos ^{2}(\theta) \mid \theta, I=1\right)=1\end{cases}
$$

By the law of large numbers, as $n \rightarrow \infty$ we have for the misfit energy (5.1)

$$
W_{n}\left(U, \varepsilon^{\mathrm{T}}\right) \rightarrow \int_{0}^{\pi / 4} g(\theta) \chi(\theta) d \rho(\theta)+(U-f)^{2}(1-f)
$$

where $g(\theta)=\left(U-f+\sin ^{2} \theta\right)^{2} \kappa(\theta)+\left(U-f+\cos ^{2} \theta\right)^{2}(1-\kappa(\theta))$ and where we have set

$$
\chi(\theta)=\mu(I=1 \mid \theta),(0 \leq \chi(\theta) \leq 1),
$$

and $f=\int_{0}^{\pi / 4} \chi(\theta) d \rho(\theta)$.

Clearly, in the present context the limiting values of the energy function $W_{n}\left(U, \varepsilon^{\mathrm{T}}\right)$ are determined uniquely by the functions $\kappa(\theta)$ and $\chi(\theta)$. Since $\mu_{\lim }$ is the limit of probability measures $\left\{\mu_{n}\right\}$ given by (4.1) with (5.1), it follows that the measure $\mu$ in (5.2) must minimize (5.4). In other words, under the assumption (5.2), the overall minimization problem has been reduced to the following minimization problem for the functions $\kappa(\theta)$ and $\chi(\theta)$ :

$$
\begin{aligned}
& \min _{\kappa(\theta), \chi(\theta)} \int_{0}^{\pi / 4} g(\theta) \chi(\theta) d \rho(\theta)+(U-f)^{2}(1-f) \\
& g(\theta)=\left(U-f+\sin ^{2} \theta\right)^{2} \kappa(\theta)+\left(U-f+\cos ^{2} \theta\right)^{2}(1-\kappa(\theta)), \\
& f=\int_{0}^{\pi / 4} \chi(\theta) d \rho(\theta), 0 \leq \chi(\theta) \leq 1,0 \leq \kappa(\theta) \leq 1 .
\end{aligned}
$$

One can anticipate that, generally, the assumption (5.2) does not hold. Indeed, even working under the assumption (5.2), we note that a solution $\mu$ (i.e., $(\kappa, \chi)$ ) to the minimization problem (5.6) may not be unique. If there are two such solutions $\mu_{1}$ and $\mu_{2}$ to this problem, then the corresponding infinite products $\tilde{\mu}_{1}$ and $\tilde{\mu}_{2}$ could, conceivably, equal to the limit of a subsequence of $\mu_{n}$. As shown in Theorem 5.2, however, in general $\mu_{\text {lim }}$ will equal a convex combination of such infinite products. The following definition will be useful in these regards.

Definition 5.1. Consider the set $\mathbb{S}$ of all measures on the set of pairs $(s, I) \in$ $[0,1] \times\{0,1\}$. For each measure $\mu \in \mathbb{S}$ define the associated product measure

$$
\tilde{\mu}=\Pi_{i=1}^{\infty} \mu
$$

on $\Pi_{i=1}^{\infty}\left(s_{i}, I_{i}\right)$. A measure $\gamma$ is called a convex combination of such product measures, if there exists a positive measure $\nu(\mu)$ on the set $\mathbb{S}$, such that

$$
\gamma(A)=\int_{\mathbb{S}} \tilde{\mu}(A) d \nu(\mu), \int_{\mathbb{S}} d \nu(\mu)=1
$$

THEOREM 5.2. Consider infinitely long grains and an arbitrary i.i.d. angle distribution with the probability measure $\rho$, and assume the limit $\mu_{\mathrm{lim}}$ of the sequence

Copyright (c) by SIAM. Unauthorized reproduction of this article is prohibited. 
$\mu_{n}$ defined by (4.2) and (4.1) exists. Then $\mu_{\mathrm{lim}}$ is given by a convex combination of product measures arising from minimization problems. In detail, we have

$$
\mu_{\lim }=\int_{\mathbb{S}} \tilde{\mu} d \nu(\mu), \int_{\mathbb{S}} d \nu(\mu)=1,
$$

where $\mathbb{S}$ is the set of product measures: $\tilde{\mu}=\prod_{i=1}^{\infty} \mu$, and each $\mu$ is defined by

$$
\mu((s, I) \mid \theta)= \begin{cases}\chi(\theta) \kappa(\theta), & \text { if } I=1, \\ 1-\chi(\theta), & \text { if } I=0,\end{cases}
$$

where $\kappa(\theta)$ and $\chi(\theta)$ are minimizers of

$$
\begin{gathered}
\min _{\kappa(\theta), \chi(\theta)} \int_{0}^{\pi / 4}(U-f+s(\theta))^{2} \chi(\theta) d \rho(\theta)+(U-f)^{2}(1-f), \\
f=\int_{0}^{\pi / 4} \chi(\theta) d \rho(\theta), \quad \chi:[0, \pi / 4] \rightarrow[0,1], \\
s(\theta)=\kappa(\theta) \sin ^{2}(\theta)+(1-\kappa(\theta)) \cos ^{2}(\theta), \quad \text { where } \kappa:[0, \pi / 4] \rightarrow\{0,1\} .
\end{gathered}
$$

If the minimizer of (5.8) is unique, then transformation strains in different grains are independent identically distributed; that is, $\mu_{\lim }$ is a product measure $\mu_{\lim }=\prod_{i=1}^{\infty} \mu$.

Proof. A key property of the energy $W_{n}$ (5.1) in the case of infinitely long grains is that it is invariant under permutations; e.g., for a three-grain polycrystal, if $\left(s_{1}, I_{1}\right),\left(s_{2}, I_{2}\right),\left(s_{3}, I_{3}\right)$ is a minimizing sequence for the angles $\left(\theta_{1}, \theta_{2}, \theta_{3}\right)$, then $\left(s_{2}, I_{2}\right),\left(s_{1}, I_{1}\right),\left(s_{3}, I_{3}\right)$ is a minimizing sequence for the angles $\left(\theta_{2}, \theta_{1}, \theta_{3}\right)$ with the same probability. More generally, the form of the misfit energy and our assumption (4.1) imply that the probability measure $\mu_{n}$ (defined by (4.1), (4.2)) on the minimizers must be symmetric ${ }^{5}$ that is, for any finite permutation $\tau \in S(n)$

$$
\mu_{n}\left(\left(s_{1}, I_{1}\right) \in A_{1} \ldots,\left(s_{n}, I_{n}\right) \in A_{n}\right)=\mu_{n}\left(\left(s_{\tau(1)}, I_{\tau(1)}\right) \in A_{1}, \ldots,\left(s_{\tau(n)}, I_{\tau(n)}\right) \in A_{n}\right) .
$$

As $n \rightarrow \infty$, the probability measure $\mu_{n}$ converges to a certain $\mu_{\text {lim }}$. Clearly, $\mu_{\text {lim }}$ must be symmetric as well: For any $n$ and $\tau \in S(n)$

$$
\begin{gathered}
\mu_{\lim }\left(\left(s_{1}, I_{1}\right) \in A_{1}, \ldots,\left(s_{n}, I_{n}\right) \in A_{n},\left(s_{n+1}, I_{n+1}\right) \in A_{n+1}, \ldots\right) \\
=\mu_{\lim }\left(\left(s_{\tau(1)}, I_{\tau(1)}\right) \in A_{1}, \ldots,\left(s_{\tau(n)}, I_{\tau(n)}\right) \in A_{n},\left(s_{n+1}, I_{n+1}\right) \in A_{n+1}, \ldots\right) .
\end{gathered}
$$

Hence, we can apply the de Finetti's representation theorem [13], and $\mu_{\text {lim }}$ must be a convex combination of product measures $\tilde{\mu}$ :

$$
\mu_{\lim }=\int_{\mathbb{S}} \tilde{\mu} d \nu(\mu), \int_{\mathbb{S}} d \nu(\mu)=1, \nu(\mu) \geq 0
$$

see Definition 5.1, as claimed.

Let us now show that

$$
\begin{aligned}
& \lim _{n \rightarrow \infty} \int_{\Omega_{n}^{\mathrm{T}}} W_{n}\left(U, \varepsilon^{\mathrm{T}}\right) d \mu_{n} \\
& \quad=\int_{\mathbb{S}}\left[\int_{0}^{\pi / 4}(U-f+s(\theta))^{2} \chi(\theta) d \rho(\theta)+(U-f)^{2}(1-f)\right] d \nu(\mu) .
\end{aligned}
$$

\footnotetext{
${ }^{5}$ Sometimes the term exchangeable is used instead of symmetric.
}

Copyright $@$ by SIAM. Unauthorized reproduction of this article is prohibited. 
The key issue here is classical: Given that $\mu_{n} \rightarrow \mu_{\text {lim }}$ weakly, we cannot, in general, conclude convergence of $\int W_{n} d \mu_{n}$. In our case, however, we can, because the measures $\mu_{n}$ are symmetric. It follows that for an $n$-grain sample, the functions $\left\{\left(s_{i}, I_{i}\right)\right\}_{i=1}^{n}$ satisfy, for any $i, j, 1 \leq i, j \leq n$,

$$
\int I_{i} I_{j} d \mu_{n}=\left\{\begin{array}{l}
\int I_{1} I_{2} d \mu_{n}, i \neq j, \\
\int I_{1}^{2} d \mu_{n}, i=j,
\end{array}\right.
$$

and similar equalities hold for $s_{i} I_{j}$, and $\left(U-\langle I\rangle+s_{i}\right)^{2}$. Therefore, $W_{n}$ can be written as

$$
\int W_{n}\left(U, \varepsilon^{\mathrm{T}}\right) d \mu_{n}=\int F\left(U, s_{1}, I_{1}, I_{2}\right) d \mu_{n}+\frac{1}{n} \int G\left(s_{1}, I_{1}, I_{2}\right) d \mu_{n},
$$

where both functions $F\left(U, s_{1}, I_{1}, I_{2}\right)$ and $G\left(s_{1}, I_{1}, I_{2}\right)$ depend continuously (they are quadratic polynomials) on the values of $s_{i}$ and $I_{i}$ only in two grains $i=1,2$ (and, thus, do not depend on $n$ ), and they are explicitly given as

$$
F=\left(U+s_{1}\right)^{2}-2 U I_{1}-2 s_{1} I_{2}+I_{1} I_{2}, \quad G=-2 s_{1} I_{1}+2 s_{1} I_{2}+I_{1}^{2}-I_{1} I_{2} .
$$

Therefore

$$
\begin{aligned}
\lim _{n \rightarrow \infty} \int_{\Omega_{n}^{\mathrm{T}}} W_{n}\left(U, \varepsilon^{\mathrm{T}}\right) d \mu_{n} & =\lim _{n \rightarrow \infty} \int_{\Omega_{n}^{\mathrm{T}}} F\left(U, s_{1}, I_{1}, I_{2}\right) d \mu_{n}+\lim _{n \rightarrow \infty} \frac{1}{n} \int_{\Omega_{n}^{\mathrm{T}}} G\left(s_{1}, I_{1}, I_{2}\right) d \mu_{n} \\
& =\int_{\Omega_{\infty}^{\mathrm{T}}} F\left(U, s_{1}, I_{1}, I_{2}\right) d \mu_{\mathrm{lim}} .
\end{aligned}
$$

Hence it only remains to show that

$$
\begin{array}{rl}
\int_{\Omega_{\infty}^{\mathrm{T}}} & F\left(U, s_{1}, I_{1}, I_{2}\right) d \mu_{\lim } \\
\quad=\int_{\mathbb{S}}\left[\int_{0}^{\pi / 4}(U-f+s(\theta))^{2} \chi(\theta) d \rho(\theta)+(U-f)^{2}(1-f)\right] d \nu(\mu) .
\end{array}
$$

The last equality is obtained by explicit computations provided in Appendix B.1. The proof of the identity (5.10) is now complete.

Further, up to a set of $\nu$-measure zero, each $\mu$ must minimize (5.8). Otherwise, we can choose a $\delta>0$ such that the set

$$
A=\left\{\mu: \int F\left(U, s_{1}, I_{1}, I_{2}\right) d \tilde{\mu}-\delta>\min \int F\left(U, s_{1}, I_{1}, I_{2}\right) d \tilde{\mu}\right\}
$$

has positive measure: $\nu(A)>0$. Then, if $\nu(\mathbb{S} \backslash A) \neq 0$ we define a new measure $\tilde{\nu}$ by

$$
\tilde{\nu}(B)=\nu(B \backslash A) / \nu(\mathbb{S} \backslash A) .
$$

Clearly

$$
\int_{\mathbb{S}}\left[\int F\left(U, s_{1}, I_{1}, I_{2}\right) d \tilde{\mu}\right] d \tilde{\nu}(\mu)<\int_{\mathbb{S}}\left[\int F\left(U, s_{1}, I_{1}, I_{2}\right) d \tilde{\mu}\right] d \nu(\mu),
$$

which contradicts the assumption that $\nu$ yields a limit of minimizers of the misfit energy (5.8) as indicated in (5.11). If $\nu(\mathbb{S} \backslash A) \neq 0$, in turn, we can select a single minimizer and assign $\tilde{\nu}$ measure 1 to it, arriving again to a contradiction.

Copyright (c) by SIAM. Unauthorized reproduction of this article is prohibited. 
To establish (5.9), note that for a minimizer $\mu$ of (5.6), the $\mu$ probabilities conditional to a given angle $\theta$ and to $I=1$, satisfy

$$
\mu\left(s(\theta)=\cos ^{2}(\theta) \mid \theta, I=1\right)=0, \text { or } \mu\left(s(\theta)=\sin ^{2}(\theta) \mid \theta, I=1\right)=0 .
$$

Hence $\kappa(\theta)$ takes only the values 0 and 1 and (5.9) holds.

Finally, suppose (5.8) admits a unique minimizer $\mu$. Since, as established above, the limit $\mu_{\text {lim }}$ must be a convex combination of product measures that minimize (5.8); in the case of uniqueness of solution to (5.8), $\mu_{\text {lim }}$ must be the product measure $\mu_{\lim }=\Pi_{i=1}^{\infty} \mu$, as claimed.

A few remarks about Theorem 5.2 are in order. Firstly, we are aware of some examples when the minimization problem (5.8) has more than one solution. One of these examples is to consider a deterministic sequence $\theta_{i}=0$ and $U=1$. Then there are two solutions $\kappa_{1} \equiv 0, \chi_{1} \equiv 1$, and $\kappa_{2} \equiv 0, \chi_{2} \equiv 0$ to the minimization problem (5.8), which give rise to corresponding measures $\mu_{1}$ and $\mu_{2}$, and, thus, product measures $\tilde{\mu}_{1}$ and $\tilde{\mu}_{2}$. For both $i=1,2$ we have

$$
\int_{0}^{\pi / 4}\left(U-f_{i}+s_{i}(\theta)\right)^{2} \chi_{i}(\theta) d \rho(\theta)+\left(U-f_{i}\right)^{2}\left(1-f_{i}\right)=1 .
$$

In view of our symmetrization assumption 4.1, the limit of $\mu_{n}$ exists and it is equal to a convex combination of product measures as implied by Theorem (5.2); the convex combination is given by $\tilde{\mu}_{1} / 2+\tilde{\mu}_{2} / 2$. We expect that generically the minimization problem (5.8) has a unique solution. We give two explicit examples when this measure is unique: Bernoulli trials, Lemma 5.3, and Uniform distribution, Lemma 5.6, in section 5.2 .

Secondly, in principle, the (unique) solution to the minimization problem (5.8) may be such that $\chi(\theta)$ takes only two values 0 or 1 , i.e., $\chi:[0, \pi / 4] \rightarrow\{0,1\}$. This, indeed, happens for uniform distribution (Lemma 5.6 in section 5.2). If we know that $\chi:[0, \pi / 4] \rightarrow\{0,1\}$, then the proof of Theorem 5.2 becomes straightforward. However, there are examples, when $0<\chi(\theta)<1$ for some $\theta$, and one of them is the Bernoulli trials (Corollary 5.4 in section 5.2).

Finally, if we do not assume the uniform conditional probability (4.1), then $\mu_{\lim }$ may not be unique even if the minimizer of (5.8) is unique. We discuss this issue for Bernoulli trials after the proof of Corollary 5.4 below.

Motivated by the above remarks, we next investigate in more detail how measure $\mu$, a solution to the minimization problem (5.8), depends on the underlying probability measure $\rho$ for two specific probability measures $\rho$ : the Bernoulli trials model and the uniform distribution of $\theta$.

5.2. Bernoulli trials and uniform distribution. For the Bernoulli trials model (4.3), the minimization problem (5.8) from Theorem 5.2 is

$$
\begin{aligned}
& \min _{q_{\alpha}, q_{\beta}, s(\alpha), s(\beta)} W\left(q_{\alpha}, q_{\beta}, s(\alpha), s(\beta)\right), \\
& W=(U-f+s(\alpha))^{2} q_{\alpha}+(U-f+s(\beta))^{2} q_{\beta}+(U-f)^{2}(1-f),
\end{aligned}
$$

with $f=q_{\alpha}+q_{\beta}$,

$$
0 \leq q_{\alpha} \leq q, 0 \leq q_{\beta} \leq 1-q,
$$

$0 \leq \beta \leq \alpha \leq \pi / 4$, and $s(\alpha), s(\beta)$ are defined by $s(\theta)=\sin ^{2} \theta$ or $s(\theta)=\cos ^{2} \theta$. In particular, the minimization with respect to $\chi$ is reduced to determining the proportions $q_{\alpha}$ and $q_{\beta}$ of grains with angles $\alpha$ and $\beta$ that do not undergo a stress-free transformation. 
Lemma 5.3. For the Bernoulli trials model (4.3) with $0<q<1$ the minimizer $(\kappa, \chi)$ of $(5.12)$ is unique. For the minimizer $\kappa(\theta) \equiv 1$, i.e.,

$$
s(\theta)=\sin ^{2} \theta,
$$

and $\chi$ depends on $U$ and can be described as follows. For a given $U$ the total proportion of grains that undergoes a stress-free transformation $f=f(U)$ is a (deterministic) nondecreasing function of $U$. For a given $f$ we have several cases

- if $f<1-q$, then

$$
q_{\alpha}=0,0 \leq q_{\beta} \leq 1-q
$$

i.e., $\chi(\alpha)=0, \chi(\beta)=q_{\beta} /(1-q)$,

- if $f>1-q$, then

$$
0 \leq q_{\alpha} \leq q, q_{\beta}=1-q,
$$

i.e. $\chi(\beta)=q_{\alpha} / q, \chi(\beta)=1$.

A proof of the Lemma is in Appendix B.2.

COROLlary 5.4. The probability distribution

$$
\theta_{i}= \begin{cases}\pi / 4, & \text { with probability } q, q \geq 1 / 2 \\ 0, & \text { with probability } 1-q\end{cases}
$$

is an example, where the minimizer $(\kappa, \chi)$ of $(5.12)$ is unique, but

$$
\chi:[0, \pi / 4] \rightarrow K \subset[0,1], K \neq\{0,1\} .
$$

Indeed, in this case

$$
\begin{aligned}
& \chi(0)=U, \chi(\pi / 4)=0, \text { if } U \leq 1-q, \\
& \chi(0)=1, \chi(\pi / 4)=0, \text { if } 1-q \leq U \leq 5 / 4-q, \\
& \chi(0)=1, \chi(\pi / 4)=U-5 / 4+q, \text { if } 5 / 4-q \leq U \leq 3 / 4, \text { or } \\
& \chi(0)=1, \chi(\pi / 4)=1, \text { if } 3 / 4 \leq U .
\end{aligned}
$$

Let us now discuss our final remark that if we do not assume the uniform conditional probability (4.1), then $\mu_{\lim }$ may not be unique even if the minimizer of (5.12) is unique. It depends on whether $\chi$ takes more than two values, that is, on whether $\chi:[0, \pi / 4] \rightarrow K$, but $K \neq\{0,1\}$. For example, consider the Bernoulli trials with $0<q_{\alpha}<q$. Then one can choose the grains with $\theta_{i}=\alpha$, which do not undergo a stress-free transformation, arbitrarily, provided that their total proportion is $q_{\alpha}$. Thus, if we remove our assumption of equal probability (4.1), in the case of infinitely long grains there are many minimizers of the misfit energy in addition to minimizers described in Theorem 5.2. Hence, it is possible to construct the limiting measure $\mu_{\text {lim }}$ on the infinite sequence of pairs $\left\{\left(s_{i}, I_{i}\right)\right\}_{i=1}^{\infty}$ so that it is not a product measure. Moreover, actual construction of the exact minimizers $\varepsilon^{\mathrm{T}}$ of the energy (5.1) (for a given sequence $\left.\left\{\theta_{i}\right\}_{i=1}^{n}\right)$ in practice $[9,6,7,8]$ is done numerically. Thus it typically results in finding an almost minimizer $\tilde{\varepsilon}^{\mathrm{T}}$, such that

$$
\left|W_{n}\left(U, \varepsilon^{\mathrm{T}}\right)-W_{n}\left(U, \tilde{\varepsilon}^{\mathrm{T}}\right)\right| \leq \delta, \delta>0 .
$$

Thus, it is natural to ask which characteristic properties of exact minimizers are approximated by characteristic properties of almost minimizers. The property 
that $\mu_{\lim }$ is a product measure is not characteristic, but the proportion of grains that undergo a stress-free transformation is such property. For example, for Bernoulli trials, $q_{\alpha}(\boldsymbol{\theta})$ characterizes the proportion of grains with $\theta_{i}=\alpha, i=1, \ldots, n$, which undergo a stress-free transformation (grains for which $I=1$ ), and we have the following immediate result.

LEMMA 5.5. For every $\delta^{\prime}>0$ there exists $\delta>0$ such that an almost minimizer $\tilde{\boldsymbol{\varepsilon}}^{T}$ in the sense (5.17) satisfies

$$
\left|q_{\alpha}(\boldsymbol{\theta})-\tilde{q}_{\alpha}(\boldsymbol{\theta})\right|<\delta^{\prime},\left|q_{\beta}(\boldsymbol{\theta})-\tilde{q}_{\beta}(\boldsymbol{\theta})\right|<\delta^{\prime},
$$

where $q_{\alpha}(\boldsymbol{\theta})$ and $\tilde{q}_{\alpha}(\boldsymbol{\theta})$ correspond to the exact and almost minimizers, respectively $\left(q_{\beta}(\boldsymbol{\theta})\right.$ and $\tilde{q}_{\beta}(\boldsymbol{\theta})$ are defined analogously).

Analogous to the Bernoulli trials model, direct computations show the following result for the uniform distribution.

LEMma 5.6. For the uniform distribution of $\theta \in[0, \pi / 4]$, the minimizer $(\kappa, \chi)$ of (5.12) is unique. For the minimizer $\kappa(\theta) \equiv 1$, hence

$$
s(\theta)=\sin ^{2} \theta,
$$

and $\chi$ depends on $U$ and can be described as follows. For a given $U$ the total proportion of grains that undergoes a stress-free transformation $f(U)$ is a (deterministic) nondecreasing function of $U$ given by

$$
f(U)=\left\{\begin{array}{l}
\frac{4 \pi}{8+\pi} U+g(U), \text { if } U \leq \frac{1}{4}+\frac{2}{\pi} \approx .88662, \\
1 \text { otherwise, }
\end{array}\right.
$$

where the small correction $g(U)$ is concave and it satisfies $g(0)=f(1 / 4+2 / \pi)=$ $0,-0.055<g(U) \leq 0$. For a given $f<1$

$$
\chi(\theta)= \begin{cases}1, & \text { if } \sin ^{2} \theta \leq 2(f-U), \\ 0, & \text { otherwise. }\end{cases}
$$

If $f=1$, then $\chi \equiv 1$.

Lemmas 5.3 and 5.6 together with Theorem 5.2 imply the following.

Corollary 5.7. For Bernoulli trials and uniform distribution, the unique minimizing sequence of transformation strains is i.i.d, and, in particular, it is uncorrelated.

6. Statistics of asymptotic energy minimizers 2: Thin long grains (finite $L \gg 1$ ).

6.1. Basic definitions and formulation of the main theorem. In contrast to the case of infinitely long grains, if $L$ is large but finite, then each grain may undergo a stress-free transformation which, as we show in this section, is correlated to stress-free transformations of other grains. In particular, for Bernoulli trials in case $1(L=\infty, n \rightarrow \infty)$ the minimizers are shown to be i.i.d. (see Corollary 5.7 in the previous section), whereas in case $2(n \rightarrow \infty$, followed by $L \rightarrow \infty)$ the minimizers are no longer i.i.d. (see Theorem 6.4 below). ${ }^{6}$

By Lemma 3.1 the misfit energy for $L \gg 1$ has the following asymptotic representation (up to higher order terms):

$$
W_{n}\left(U, \varepsilon^{\mathrm{T}}\right)=\left\langle(U-f+s)^{2}\right\rangle-\frac{B}{L} \bar{W}_{n}\left(U, \varepsilon^{\mathrm{T}}\right), \text { as } L \rightarrow \infty
$$

\footnotetext{
${ }^{6}$ In this sense, we prove that the limits for large $n$ and large $L$ do not commute.
} 
where

$$
\begin{gathered}
\bar{W}_{n}\left(U, \varepsilon^{\mathrm{T}}\right)=\int_{-1}^{1} \int_{-1}^{1}(s(x)-\langle s\rangle)(s(t)-\langle s\rangle) K_{\infty}(x-t) d x d t, \\
K_{\infty}=-\ln |\sin (\pi x / 2)|>0 .
\end{gathered}
$$

For $L \gg 1$ the second term on the right-hand side of (6.1) amounts to a small correction to the misfit energy of infinitely long grains (5.1) (the first term in (6.1)), a situation that bears connections with the concept of almost minimizers (5.17) introduced in the previous section. In the present context we have:

Lemma 6.1. For any $\delta>0$, there exists a sufficiently large $L_{0}>0$, so that for any $L \geq L_{0}$

$$
\begin{gathered}
s(\theta)=\sin ^{2} \theta, \\
\left|q_{\alpha}(\boldsymbol{\theta})-q_{\alpha}^{L}(\boldsymbol{\theta})\right|<\delta,\left|q_{\beta}(\boldsymbol{\theta})-q_{\beta}^{L}(\boldsymbol{\theta})\right|<\delta,
\end{gathered}
$$

where $q_{\alpha}(\boldsymbol{\theta})$ and $q_{\alpha}^{L}(\boldsymbol{\theta})$ correspond to minimizers of (5.1) and (6.1), respectively $\left(q_{\beta}(\boldsymbol{\theta})\right.$ and $q_{\beta}^{L}(\boldsymbol{\theta})$ are defined analogously).

A proof of Lemma 6.1 is in Appendix C.1. As we pointed out after Corollary 5.4, when $L=\infty$ and $0<q_{\alpha}<q$ or $0<q_{\beta}<1-q$ there is an ambiguity: The solution to the minimization problem (5.1) (the first term in (6.1)) is not unique. We now show that the second term (6.2) plays a role of regularization-it resolves this ambiguity by reducing the number of minimizers, and it gives rise to correlation of transformations in different grains of a laminated polycrystal. The correlations arise from maximization of $\bar{W}$ (note the negative sign in front of the second term in (6.1)). We formalize this idea in the next definition.

DEFINITION 6.2. For a given deformation $U$ and a sequence of angles $\left\{\theta_{i}\right\}_{i=1}^{n}$, an asymptotic energy minimizer is a pair of piecewise constant functions

$$
(s(x), I(x))=\left(s_{i}, I_{i}\right) \text { if } x \in\left(\frac{i-1}{n}, \frac{i}{n}\right], i=1,2, \ldots, n, s(x), I(x) \in \mathcal{H}_{n},
$$

which maximizes (6.2) (the second term in (6.1)) amongst all minimizers of the misfit energy of infinitely long grains (5.1) (the first term in (6.1)).

When the distribution of angles $\rho_{n}$ is given, we denote by $\mu_{n}^{a}$ the corresponding probability measure of the distribution of asymptotic energy minimizers $\left(s_{i}, I_{i}\right)_{i=1}^{n}$. The behavior of the asymptotic energy minimizers for the distribution of angles $\rho_{n}$ given by the Bernoulli trials (4.3) model will be described by the Riesz symmetrically rearranged minimizer which we define as follows.

Definition 6.3. Consider Bernoulli trials (4.3) model ( $\theta$ is $\alpha$ or $\beta$ with probabilities $q$ or $1-q)$. For a given displacement $U$, let $q_{\alpha}$ and $q_{\beta}$ be the proportion of grains for which $I=1$ with $\theta=\alpha$ and $\theta=\beta$, respectively,

$$
0 \leq q_{\alpha} \leq q, \quad 0 \leq q_{\beta} \leq 1-q,
$$

\footnotetext{
${ }^{7}$ Note that we consider here all possible minimizers of the misfit energy of infinitely long grains (5.1) (the first term in (6.1)). In other words, here minimizers of (5.1) may not satisfy our assumption of equal probability (4.1).
}

Copyright (c) by SIAM. Unauthorized reproduction of this article is prohibited. 
as given by Lemma 5.3. For a given sequence of angles $\left\{\theta_{i}\right\}_{i=1}^{n}$ a Riesz left-rearranged sequence of transformation strains is a pair of functions $\left(s_{l}(x), I_{l}(x)\right) \in \mathcal{H}_{n}$ given for each $x \in((i-1) / n, i / n]$ as

$\left(s_{l}(x), I_{l}(x)\right)=\left(s_{l}\left(\frac{i}{n}\right), I_{l}\left(\frac{i}{n}\right)\right)=\left\{\begin{array}{l}\left(\sin ^{2} \alpha, 1\right), \text { if } \theta=\alpha, 0 \leq i / n \leq q_{\alpha} / q, \\ (0,0), \text { if } \theta=\alpha, q_{\alpha} / q \leq i / n \leq 1, \\ \left(\sin ^{2} \beta, 1\right), \text { if } \theta=\beta, 0 \leq i / n \leq q_{\beta} /(1-q), \\ (0,0), \text { if } \theta=\beta, q_{\beta} /(1-q) \leq i / n \leq 1 .\end{array}\right.$

Similarly, a Riesz right-rearranged sequence $\left(s_{r}(x), I_{r}(x)\right) \in \mathcal{H}_{n}$ is determined by

$$
\begin{aligned}
\left(s_{r}(x), I_{r}(x)\right)= & \left(s_{r}\left(\frac{i}{n}\right), I_{r}\left(\frac{i}{n}\right)\right) \\
= & \left\{\begin{array}{l}
\left(\sin ^{2} \alpha, 1\right), \text { if } \theta=\alpha, 1-q_{\alpha} / q \leq i / n \leq 1, \\
(0,0), \text { if } \theta=\alpha, 0 \leq i / n \leq 1-q_{\alpha} / q, \\
\left(\sin ^{2} \beta, 1\right), \text { if } \theta=\beta, 1-q_{\beta} /(1-q) \leq i / n \leq 1, \\
(0,0), \text { if } \theta=\beta, 0 \leq i / n \leq 1-q_{\beta} /(1-q) .
\end{array}\right.
\end{aligned}
$$

We denote by $\mu_{n}^{l}$ and $\mu_{n}^{r}$ the corresponding probability measures. A Riesz symmetrically rearranged probability measure $\mu_{n}^{s}$ is the average of right- and left-rearranged measures $\mu_{n}^{s}=1 / 2 \mu_{n}^{l}+1 / 2 \mu_{n}^{r}$. Finally, the Riesz symmetrically rearranged probability measure $\mu^{s}$, the weak limit of probability measures: $\mu_{n}^{s} \Rightarrow \mu^{s}$, as $n \rightarrow \infty$.

The rearranged minimizers quantitatively describe the rise of correlations for asymptotic energy minimizers (Definition 6.2), because, as we prove in Lemma 6.7, the asymptotic and rearranged minimizers coincide in the limit as $n \rightarrow \infty$. In other words, Definitions 6.2 and 6.3 characterize the same measure as $n \rightarrow \infty$. Moreover, the following theorem shows that the minimizer probability measure of the full misfit energy (6.1) converges to the Riesz symmetrically rearranged probability measure when we let $n \rightarrow \infty$, and then let $L \rightarrow \infty$.

Theorem 6.4. Consider the Bernoulli trials (4.3). For a given $U$, let $q_{\alpha}$ and $q_{\beta}$ be defined as in Lemma 5.3 by (5.15) or (5.16). Then as $n \rightarrow \infty$ and subsequently $L \rightarrow \infty$ the probability measure of the energy minimizer of the misfit energy (6.1) converges weakly to the Riesz symmetrically rearranged probability measure $\mu^{s}$.

6.2. Riesz rearrangement inequalities and proof of Theorem 6.4. The key idea of the proof comes from the classical Riesz rearrangement inequality (see e.g., [12], [14]). In particular, this inequality motivated the name for minimizers in Definition 6.3. The simplest form of this inequality, which is sufficient for our purposes is as follows.

LEMma 6.5. Riesz rearrangement inequality on a circle.

Consider two classes of even, bounded, and positive functions on $[-1,1]$ :

$$
\mathcal{A}_{i}=\left\{f(x) \mid f(x)=f(-x), 0 \leq f(x) \leq 1, \int_{0}^{1} f(x) d x=p_{i}\right\}, i=1,2 .
$$

Let $\chi_{p_{i}}^{1}(x) \in \mathcal{A}_{i}$ be the characteristic function of the set $\left[-p_{i}, p_{i}\right]$ and $\chi_{p_{i}}^{2}(x) \in \mathcal{A}_{i}$ be the characteristic function of the set $\left[-1,-1+p_{i}\right] \cup\left[1-p_{i}, 1\right]$. Suppose $K(x)$ is an even positive locally integrable 2 -periodic function on $\mathbb{R}$ that decreases on $[0,1]$ :

$K(x) \geq 0, K(x)=K(-x), \int_{0}^{1} K(x) d x<\infty, K(x+2)=K(x)$, and $K\left(x_{1}\right)>K\left(x_{2}\right)$, 
if $0<x_{1}<x_{2} \leq 1$. Then for any $f(x) \in \mathcal{A}_{1}, g(x) \in \mathcal{A}_{2}$

$$
\begin{aligned}
& \int_{-1}^{1} \int_{-1}^{1}\left(f(x)-p_{1}\right)\left(g(t)-p_{2}\right) K(x-t) d x d t \\
& \quad \leq \int_{-1}^{1} \int_{-1}^{1}\left(\chi_{p_{1}}^{1}(x)-p_{1}\right)\left(\chi_{p_{2}}^{1}(t)-p_{2}\right) K(x-t) d x d t,
\end{aligned}
$$

where the equality holds only in the following two cases

$$
\text { (a) } f(x)=\chi_{p_{1}}^{1}(x), g(x)=\chi_{p_{2}}^{1}(x),(b) f(x)=\chi_{p_{1}}^{2}(x), g(x)=\chi_{p_{2}}^{2}(x) .
$$

Moreover, for any $\delta>0$ there exists $\delta^{\prime}>0$ so that if $\min \left(e_{1}, e_{2}\right) \geq \delta^{\prime}$,

$$
e_{1}=\int_{0}^{1}\left(\left|f-\chi_{p_{1}}^{1}\right|+\left|g-\chi_{p_{2}}^{1}\right|\right) d x, e_{2}=\int_{0}^{1}\left(\left|f-\chi_{p_{1}}^{2}\right|+\left|g-\chi_{p_{2}}^{2}\right|\right) d x,
$$

then

$$
\begin{aligned}
& \int_{-1}^{1} \int_{-1}^{1}\left(f(x)-p_{1}\right)\left(g(t)-p_{2}\right) K(x-t) d x d t \\
& \quad \leq \int_{-1}^{1} \int_{-1}^{1}\left(\chi_{p_{1}}^{1}(x)-p_{1}\right)\left(\chi_{p_{2}}^{1}(t)-p_{2}\right) K(x-t) d x d t-\delta .
\end{aligned}
$$

We assumed in this lemma that the functions are bounded from above by one. This assumption can be replaced by any positive number with obvious modifications of the results. The proof of Lemma 6.5 follows from considerations similar to those found in [1]. It basically says that among all possible functions $0 \leq f(x) \leq 1,0 \leq g(x) \leq 1$ on a circle $[-1,1]$ (where the endpoints $x= \pm 1$ are identified) the maximum of the integral

$$
\int_{-1}^{1} \int_{-1}^{1}\left(f(x)-p_{1}\right)\left(g(t)-p_{2}\right) d x d t, \text { with } \int_{0}^{1} f(x) d x=p_{1}, \int_{0}^{1} g(x) d x=p_{2}
$$

is achieved on characteristic functions of the intervals of length $2 p_{1}$ and $2 p_{2}$. The reason why the intervals centered at $x=0$ and $x=1$ is due to our assumption that $f(x)$ and $g(x)$ are even. In order to explain how Lemma 6.5 must be modified and applied for our case, we decompose

$$
s(x)-\langle s\rangle=\sin ^{2} \alpha \varepsilon_{\alpha}+\sin ^{2} \beta \varepsilon_{\beta}, \varepsilon_{\alpha}=\left(\chi_{\alpha} \tilde{\chi}_{\alpha}-q_{\alpha}\right), \varepsilon_{\beta}=\left(\chi_{\beta} \tilde{\chi}_{\beta}-q_{\beta}\right),
$$

where $\chi_{\alpha}$ and $\chi_{\beta}, \chi_{\alpha}+\chi_{\beta}=1$ are (random) characteristic functions of the angle distributions $\theta=\alpha$ and $\theta=\beta$, respectively; $\tilde{\chi}_{\alpha}$ and $\tilde{\chi}_{\beta}$ are the characteristic function of the grains with $\theta=\alpha$ and $\theta=\beta$, respectively, for which $I=1$.

The term (6.2) (the second term in (6.1)) equals

$$
\begin{aligned}
& \bar{W}\left(U, \varepsilon^{\mathrm{T}}\right)=\sin ^{4} \alpha \int_{-1}^{1} \int_{-1}^{1} \varepsilon_{\alpha}(x) \varepsilon_{\alpha}(t) K_{\infty}(x-t) d x d t \\
& +2 \sin ^{2} \alpha \sin ^{2} \beta \int_{-1}^{1} \int_{-1}^{1} \varepsilon_{\alpha}(x) \varepsilon_{\beta}(t) K_{\infty}(x-t) d x d t \\
& +\sin ^{4} \beta \int_{-1}^{1} \int_{-1}^{1} \varepsilon_{\beta}(x) \varepsilon_{\beta}(t) K_{\infty}(x-t) d x d t .
\end{aligned}
$$

Copyright $@$ by SIAM. Unauthorized reproduction of this article is prohibited. 

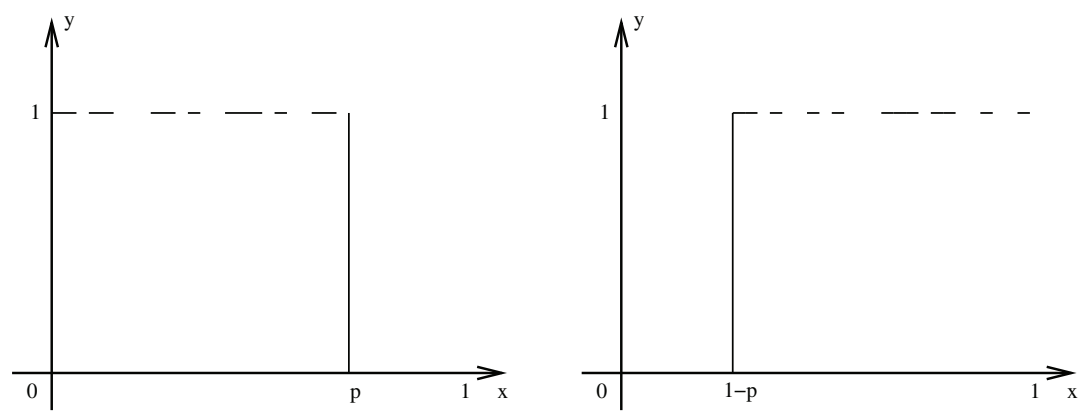

FIG. 6.1. A sample of two random intervals $\chi_{\alpha}(x) \chi_{q_{\alpha}}^{1}(x)$ and $\chi_{\alpha}(x) \chi_{q_{\alpha}}^{2}(x)$, where $p=q_{\alpha} / q$.

Each of the three integral terms in (6.13) has the form described in the previous Lemma 6.5 , because by Lemma 3.1 the integral kernel $K_{\infty}=-\ln |\sin (\pi x / 2)|$, and hence it satisfies all the conditions (6.7). As in Lemma 6.5 we need to maximize the integral (6.13) by varying the characteristic functions $\tilde{\chi}_{\alpha}$ and $\tilde{\chi}_{\beta}$. The only difference is the additional constraint that $\chi_{\alpha}$ and $\chi_{\beta}$ are random characteristic functions. This additional constraint, loosely speaking, requires that the maximizers of (6.13) are "random intervals" still centered at $x=0$ or $x=1$. More precisely, note that the values of the characteristic functions $\tilde{\chi}_{\alpha}$ and $\tilde{\chi}_{\beta}$ in (6.13) are important only where $\chi_{\alpha}=1$ and $\chi_{\beta}=1$, respectively. Hence for a sequence of Bernoulli random variables $\theta_{i}, i=1, \ldots, n$ we can define characteristic functions of random intervals of length $2 q_{\alpha}$ on $[-1,1]$ centered at $x=0$ and $x=1$ as a product of two characteristic functions $\chi_{\alpha}(x) \chi_{q_{\alpha}}^{1}(x)$ and $\chi_{\alpha}(x) \chi_{q_{\alpha}}^{2}(x)$, respectively, where

$$
\chi_{q_{\alpha}}^{1}(x)=\left\{\begin{array}{l}
1,-\frac{q_{\alpha}}{q} \leq x \leq \frac{q_{\alpha}}{q}, \\
0, \text { otherwise }
\end{array} \quad \chi_{q_{\alpha}}^{2}(x)=\left\{\begin{array}{l}
0,-1+\frac{q_{\alpha}}{q}<x<1-\frac{q_{\alpha}}{q}, \\
1, \text { otherwise }
\end{array}\right.\right.
$$

Similarly, functions $\chi_{\alpha}(x) \chi_{q_{\alpha}}^{1}(x)$ and $\chi_{\alpha}(x) \chi_{q_{\alpha}}^{2}(x)$ are random intervals of length $2 q_{\beta}$ centered at $x=0$ and $x=1$ where

$$
\chi_{q_{\beta}}^{1}(x)=\left\{\begin{array}{l}
1,-\frac{q_{\beta}}{1-q} \leq x \leq \frac{q_{\beta}}{1-q}, \\
0, \text { otherwise }
\end{array} \quad \chi_{q_{\beta}}^{2}(x)=\left\{\begin{array}{l}
0,-1+\frac{q_{\beta}}{1-q}<x<1-\frac{q_{\beta}}{1-q} \\
1, \text { otherwise }
\end{array}\right.\right.
$$

For an illustration see Figure 6.1. The above discussion is made rigorous by the following.

LEMMA 6.6 (randomized Riesz rearrangement inequality for asymptotic energy minimizers). Consider Bernoulli trials (4.3). Suppose $\chi_{\alpha}$ and $\chi_{\beta}, \chi_{\alpha}+\chi_{\beta}=1$ are (random) characteristic functions of the angle distributions $\theta=\alpha$ and $\theta=\beta$, respectively. Let $q_{\alpha}(\boldsymbol{\theta})$ and $q_{\beta}(\boldsymbol{\theta})$ be random variables of $\boldsymbol{\theta}$ with values

$$
0 \leq q_{\alpha}(\boldsymbol{\theta}) \leq q, 0 \leq q_{\beta}(\boldsymbol{\theta}) \leq 1-q
$$

Suppose $K(x)$ satisfies (6.7). Then for every $\delta>0$ there exists $\delta^{\prime}>0$ so that if

$$
\left|q_{\alpha}(\boldsymbol{\theta})-q_{\alpha}\right|<\delta^{\prime},\left|q_{\beta}(\boldsymbol{\theta})-q_{\beta}\right|<\delta^{\prime}
$$


for some fixed $q_{\alpha}$ and $q_{\beta}$, then almost surely as $n \rightarrow \infty$ the maximizers of

$$
\begin{aligned}
& \max _{\tilde{\chi}_{\alpha}, \tilde{\chi}_{\beta}} \int_{-1}^{1} \int_{-1}^{1}\left(a \varepsilon_{\alpha}(x)+b \varepsilon_{\beta}(x)\right)\left(a \varepsilon_{\alpha}(t)+b \varepsilon_{\beta}(t)\right) K(x-t) d x d t \\
& \varepsilon_{\alpha}=\left(\chi_{\alpha} \tilde{\chi}_{\alpha}-q_{\alpha}(\boldsymbol{\theta})\right), \varepsilon_{\beta}=\left(\chi_{\beta} \tilde{\chi}_{\beta}-q_{\beta}(\boldsymbol{\theta})\right), a>0, b>0 \\
& \chi^{\alpha}(x)=\chi^{\alpha}(-x), \chi^{\beta}(x)=\chi^{\beta}(-x), \quad \int_{0}^{1} \chi^{\alpha} \tilde{\chi}_{\alpha} d x=q_{\alpha}(\boldsymbol{\theta}), \quad \int_{0}^{1} \chi^{\beta} \tilde{\chi}_{\beta} d x=q_{\beta}(\boldsymbol{\theta})
\end{aligned}
$$

satisfy

$$
\min \left(e_{1}, e_{2}\right)<\delta
$$

where

$e_{1}=\int_{0}^{1}\left(\left|\tilde{\chi}_{\alpha}-\chi_{q_{\alpha}}^{1}\right| \chi_{\alpha}+\left|\tilde{\chi}_{\beta}-\chi_{q_{\beta}}^{1}\right| \chi_{\beta}\right) d x, e_{2}=\int_{0}^{1}\left(\left|\tilde{\chi}_{\alpha}-\chi_{q_{\alpha}}^{2}\right| \chi_{\alpha}+\left|\tilde{\chi}_{\beta}-\chi_{q_{\beta}}^{2}\right| \chi_{\beta}\right) d x$,

$\chi_{q_{\alpha}}^{i}$ and $\chi_{q_{\beta}}^{i}, i=1,2$ are defined in (6.14) and (6.15), respectively.

The proof of Lemma 6.6 is by contradiction to the law of large numbers and it is provided in Appendix C.2. The next lemma shows the equivalence of Definitions 6.2 and 6.3 as $n \rightarrow \infty$.

Lemma 6.7. Consider the Bernoulli trials (4.3). For a given $U$, let $q_{\alpha}$ and $q_{\beta}$ be defined as in Lemma 5.3 by (5.15) or (5.16). Then the probability measure $\mu_{n}^{a}$ of the asymptotic energy minimizer of the misfit energy (6.1) (see Definition 6.2)) and the probability measure $\mu_{n}^{s}$ of the Riesz symmetrically rearranged energy minimizer (Definition 6.3) have the same weak limit $\mu^{s}$. Moreover,

$$
\lim _{n \rightarrow \infty} e_{1}=0, \text { or } \lim _{n \rightarrow \infty} e_{2}=0 \text { with equal probability } 1 / 2,
$$

where $e_{1}$ and $e_{2}$ are defined by (6.19) in Lemma 6.17 above.

Proof. By Lemmas 5.3 and 5.5, depending on $U$, the minimizer of the first term in (6.1) satisfies (5.15) or (5.16) for almost every $\boldsymbol{\theta}$ as $n \rightarrow \infty$. Since the condition (6.16) of the randomized Riesz rearrangement inequality is satisfied for any $\delta>0$, by Lemma 6.17 for almost every $\boldsymbol{\theta}$,

$$
\lim _{n \rightarrow \infty} \min \left(e_{1}, e_{2}\right)=0
$$

Hence $\left\{\left(s_{i}, I_{i}\right)\right\}_{i=1}^{n}$ is either a left-rearranged or right-rearranged sequence almost surely as $n \rightarrow \infty$. For every $n$ the measure $\mu_{n}^{a}$ must be symmetric with respect to the to reflection about the point $x=1 / 2$, i.e. with equal probability either $e_{1} \rightarrow 0$ or $e_{2} \rightarrow 0$ as $n \rightarrow \infty$. This proves (6.20). Clearly (6.20) implies that $\mu_{n}^{a}$ and the Riesz symmetrically rearranged measure $\mu_{n}^{s}$ have the same weak limit as $n \rightarrow \infty$.

End of proof of Theorem 6.4. Again, by the symmetry of the problem with respect to reflection about the point $x=1 / 2, \mu_{n}$ also must be similarly symmetric. By Lemma 6.1 the minimizer of (6.1) is an almost minimizer, i.e. for any $\delta>0$ there is $L_{0}$ so that the condition (6.16) of the randomized Riesz rearrangement inequality holds.

\footnotetext{
${ }^{8}$ Here and in the sequel almost sure convergence is considered in the probability space $(\Omega, \mathcal{F}, P)$ set in the beginning of section 4 .
}

Copyright (C) by SIAM. Unauthorized reproduction of this article is prohibited. 
Hence it implies that for sufficiently large $L_{0}$ the minimizing sequences $\left\{\left(s_{i}, I_{i}\right)\right\}_{i=1}^{n}$ for any $L>L_{0}$ are arbitrarily close to the Riesz symmetrically rearranged minimizing sequences, namely either

$(6.21)$

$\int_{0}^{1}\left(\left|\tilde{\chi}_{\alpha}-\chi_{q_{\alpha}}^{1}\right| \chi_{\alpha}+\left|\tilde{\chi}_{\beta}-\chi_{q_{\beta}}^{1}\right| \chi_{\beta}\right) d x<\delta$, or $\int_{0}^{1}\left(\left|\tilde{\chi}_{\alpha}-\chi_{q_{\alpha}}^{2}\right| \chi_{\alpha}+\left|\tilde{\chi}_{\beta}-\chi_{q_{\beta}}^{2}\right| \chi_{\beta}\right) d x<\delta$,

with equal probability $1 / 2$ as $n \rightarrow \infty$. If $L_{0} \rightarrow \infty$, then $\delta \rightarrow 0$, and this completes the proof.

It follows from (6.21) that long/short-range correlations in the minimizing sequences $\left\{\left(s_{i}, I_{i}\right)\right\}_{i=1}^{n}$ are determined by long/short-range correlations of the Riesz symmetrically rearranged minimizing sequences. There is no short-range correlations of the Riesz symmetrically rearranged minimizing sequences. Riesz rearranged measure is, however, correlated on the large-scale: For example, suppose $q_{\alpha}=q$ and $q_{\beta}<1-q$, then for the right-rearranged measure:

$$
(s, I)=\left\{\begin{array}{l}
\left(\sin ^{2} \alpha, 1\right), \text { with probability } q \\
\left(\sin ^{2} \beta, 1\right), \text { with probability } 1-q, \text { if } 1-q_{\beta} /(1-q) \leq x \leq 1, \\
(0,0), \text { with probability } 1-q, \text { if } 0 \leq x<1-q_{\beta} /(1-q),
\end{array}\right.
$$

and for the left-rearranged measure:

$$
(s, I)=\left\{\begin{array}{l}
\left(\sin ^{2} \alpha, 1\right), \text { with probability } q \\
\left(\sin ^{2} \beta, 1\right), \text { with probability } 1-q, \text { if } 0 \leq x<q_{\beta} /(1-q), \\
(0,0), \text { with probability } 1-q, \text { if } q_{\beta} /(1-q) \leq x \leq 1 .
\end{array}\right.
$$

The long-range correlation of transformation strains for the symmetrically rearranged minimizer probability measure can be read off the formulas (6.22) and (6.23).

\section{Statistics of asymptotic energy minimizers 3: Chain of short grains.}

7.1. Basic definitions and ideas. In this section we will show how exponentially decaying correlations arise when the scaling of the polycrystal is such that $L=l_{0} /(2 n), l_{0} \ll 1$ (short grains). By estimate (3.16) the misfit energy for $l_{0} \ll 1$ is given (up to higher order terms) by the nearest neighbor energy

$$
W_{n}\left(U, \varepsilon^{\mathrm{T}}\right)=\left\langle(U-f+s)^{2}\right\rangle-\lambda_{0}\left\langle(s-\langle s\rangle)^{2}\right\rangle-\frac{B l_{0}}{n} \sum_{i=1}^{n}\left(s_{i}-\langle s\rangle\right)\left(s_{i+1}-\langle s\rangle\right)
$$

where $B>0, \lambda_{0}>0$. In this case, we show that for Bernoulli trials (4.3) exponentially decaying correlations arise when $n \rightarrow \infty$, followed by $l_{0} \rightarrow 0$.

Qualitatively, the misfit energy $W_{n}^{1}\left(U, \varepsilon^{\mathrm{T}}\right)$ has three terms which are analogous to the case of thin long grains (6.1). The minimization of the first two terms,

$$
W_{n}^{0}\left(U, \varepsilon^{\mathrm{T}}\right)=\left\langle(U-f+s)^{2}\right\rangle-\lambda_{0}\left\langle(s-\langle s\rangle)^{2}\right\rangle
$$

determines, as in Lemma $5.3, q_{\alpha}$ and $q_{\beta}$, the total amount of the grains that undergo a stress-free transformation. The minimizers of (7.2) are, in general, not unique. The third term provides a small correction to (7.2), and, as in section 6 , plays a role of regularization, that is it selects the unique minimizer of (7.2) that maximizes

$$
\bar{W}_{n}\left(U, \varepsilon^{\mathrm{T}}\right):=\frac{1}{n} \sum_{i=1}^{n}\left(s_{i}-\langle s\rangle\right)\left(s_{i+1}-\langle s\rangle\right)
$$

Copyright (c) by SIAM. Unauthorized reproduction of this article is prohibited. 
Analogous to Definition 6.2, the above considerations motivate the following definition:

Definition 7.1. For a fixed sequence $\theta_{i}, i=1, \ldots, n$, the asymptotic energy minimizer of the nearest neighbor model (7.1) is the sequence $\left(s_{i}, I_{i}\right), i=1, \ldots, n$ such that it minimizes (7.2) and maximizes (7.3) among minimizers of (7.2).

Lemma 7.2. For the Bernoulli trials model (4.3) the minimizing sequence $\left(s_{i}, I_{i}\right)$, $i=1, \ldots, n$ of the misfit energy $W_{n}^{0}\left(U, \varepsilon^{T}\right)$ given by (7.2) satisfies

$$
s(\theta)=\sin ^{2} \theta,
$$

and as $n \rightarrow \infty$,

$$
q_{\alpha}(\boldsymbol{\theta}) \rightarrow q_{\alpha}, q_{\beta}(\boldsymbol{\theta}) \rightarrow q_{\beta}
$$

almost surely. The values $q_{\alpha}$ and $q_{\beta}$ are determined as follows. For a given $U$ the total proportion of grains that undergoes a stress-free transformation $f=f(U)$ is a (deterministic) nondecreasing function of $U$. For a given $f$ we have several cases:

- if $f<1-q$, then

$$
q_{\alpha}=0,0 \leq q_{\beta} \leq 1-q,
$$

$\chi(\alpha)=0, \chi(\beta)=q_{\beta} /(1-q)$,

- if $f>1-q$, then

$$
0 \leq q_{\alpha} \leq q, q_{\beta}=1-q
$$

$$
\chi(\beta)=q_{\alpha} / q, \chi(\beta)=1 .
$$

There are values of $\alpha, \beta$, and $U$ for which $0<q_{\beta}<1-q$ or $0<q_{\alpha}<q$.

The proof of this lemma is analogous to Lemma 5.3. Clearly, the function $f(U)$ in Lemma 7.2 is different from the one for the infinitely long grains in Lemma 5.3. However, the characteristic property of the measure that it is determined by $q_{\alpha}$ and $q_{\beta}$ with either (7.5) or (7.6) still holds. One of the consequences of the previous lemma is that there are, again, some values $q_{\alpha}$ and $q_{\beta}$ that determine the proportion of grains that undergo a stress-free transformation and they satisfy $q_{\alpha}=0$ or $q_{\beta}=1-q$. This is exactly the characteristic property that we need to be able to prove exponential decay of correlations by applying the isoperimetric inequality (7.8) to the sequences described in Definition 7.1. Following the logic in section 6, we obtain that asymptotic energy minimizer of the nearest neighbor model (7.1) arises in the limit $n \rightarrow \infty$, followed by $l_{0} \rightarrow \infty$. The proof of this statement is similar to the proof of the analogous statement in case 2; see end of the proof of Theorem 6.4 in section 6.2. Hence we only need to find a statistical characterization of maximizers of (7.3) for fixed $q_{\alpha}$ and $q_{\beta}$ found from Lemma 7.2. This is given in Theorems 7.4 and 7.5 below.

7.2. Isoperimetric inequalities and characterization of maximizers of (7.3). Here, it is convenient to characterize any point in the composite $x \in[0,1]$ as a point that belongs to a (maximal) uninterrupted string of identical values of $\theta$.

DeFinition 7.3. For a fixed $\boldsymbol{\theta}=\theta_{1}, \theta_{2}, \ldots, \theta_{n}$ we say that a string

$$
\theta_{\alpha}^{m}=\theta_{i+1}, \theta_{i+2}, \ldots, \theta_{i+m}, \theta_{\alpha}^{m} \subset \boldsymbol{\theta}
$$

is a (maximal) uninterrupted string of $\theta=\alpha$ of length $m$ if all $\theta_{i+j}=\alpha, i=1, \ldots, m$ and $\theta_{i}=\theta_{i+1+m}=\beta$. We say that $x \in[0,1]$ belongs to an uninterrupted string of values $\alpha$ of length $m$ if $x \in \theta_{\alpha}^{m}$. The notion $x \in \theta_{\beta}^{m}$ is defined analogously.

Copyright $@$ by SIAM. Unauthorized reproduction of this article is prohibited. 
Recall our notation

$$
\begin{gathered}
s(x)-\langle s\rangle=\sin ^{2} \alpha \varepsilon_{\alpha}+\sin ^{2} \beta \varepsilon_{\beta}, \\
\varepsilon_{\alpha}=\left(\chi_{\alpha} \tilde{\chi}_{\alpha}-q_{\alpha}\right), \quad \varepsilon_{\beta}=\left(\chi_{\beta} \tilde{\chi}_{\beta}-q_{\beta}\right),
\end{gathered}
$$

where $\chi_{\alpha}$ and $\chi_{\beta}, \chi_{\alpha}+\chi_{\beta}=1$ are (random) characteristic functions of the angle distributions $\theta=\alpha$ and $\theta=\beta$, respectively; $\tilde{\chi}_{\alpha}$ and $\tilde{\chi}_{\beta}$ are the characteristic function of the grains with $\theta=\alpha$ and $\theta=\beta$, respectively, for which $I=1$.

By Lemma 7.2, we have two cases: Either $q_{\beta}=1-q$, and then $\tilde{\chi}_{\beta} \equiv 1$, or $q_{\alpha}=0$, and then $\tilde{\chi}_{\alpha} \equiv 0$. Let us study these two cases separately.

Suppose $q_{\alpha}=0$. Let us look at maximization of

$$
\frac{1}{n} \sum_{i=1}^{n} s_{i} s_{i+1},\langle s\rangle=q_{\beta} \sin ^{2} \beta
$$

only. Each of $s_{i}$ (up to the constant $\sin ^{2} \beta$ ) is either 1 or 0 ; therefore the maximization of the nearest neighbors term (7.7) can be understood as the minimization of the boundary of a set with constant area:

$$
\min _{D \in \mathcal{A}} \partial D, \mathcal{A}=\left\{D \mid D=\left\{x \mid s_{i}(x) \neq 0\right\}, \int_{D} d x=q_{\beta}\right\} .
$$

Then the usual isoperimetric inequality implies that the maximizer of (7.7) is such that the grains with $\theta_{i}=\beta$ undergo a stress-free transformation, if they belong to a "long" uninterrupted sequence $\theta_{\beta}^{m}$ of the grains with the same $\theta=\beta$. If $\theta_{i}=\beta$ belongs to a "short" uninterrupted sequence $\theta_{\beta}^{m}$, then it does not undergo a stress-free transformation. Hence there should be short-range correlations. The notion of short and long sequences is relative to the value of the total number of grains that must undergo a stress-free transformation. The above ideas are formulated more precisely in the next theorem.

Theorem 7.4. Consider the Bernoulli trials (4.3). Denote by $\tilde{\chi}_{\alpha}$ and $\tilde{\chi}_{\beta}$ the characteristic function of the grains with $\theta=\alpha$ and $\theta=\beta$, respectively, for which $I=1$. Suppose $U$ is such that the minimizer of the first term in (7.1) satisfies $q_{\alpha}=0$. Then in the limit $n \rightarrow \infty$ the sequence $\left(s_{i}, I_{i}\right), i=1,2, \ldots, n, \ldots$ is a stationary process with exponentially decaying short-range correlations, long-range correlations are zero, and $\tilde{\chi}_{\alpha} \equiv 1$. Moreover, almost surely as $n \rightarrow \infty$ the minimizer of the nearest neighbor model (7.1) satisfies

$$
\tilde{\chi}_{\beta}(x)=\left\{\begin{array}{l}
1, \text { if } x \in \theta_{\beta}^{m}, m>k, \\
0, \text { if } x \in \theta_{\beta}^{m}, m<k, \\
1, \text { with probability } r, \text { if } x \in \theta_{\beta}^{k}, \\
0, \text { with probability } 1-r, \text { if } x \in \theta_{\beta}^{k},
\end{array}\right.
$$

where

$$
k=\max (m) \text { such that } q_{\beta}<(1-q)^{m},
$$

and $r$ is found from

$$
q_{\beta}=r q(1-q)^{k}-(1-q)^{k+1}
$$

Copyright $@$ by SIAM. Unauthorized reproduction of this article is prohibited. 
Proof. By the law of the large numbers, $\tilde{\chi}_{\alpha}=0$ and $q_{\beta}(\boldsymbol{\theta}) \rightarrow q_{\beta}$ almost surely; therefore it is sufficient to study (7.7) or, equivalently, (7.8). By the isoperimetric inequality for every $\boldsymbol{\theta}$ the function $\tilde{\chi}_{\beta}(x)$ must be such that if $\theta_{\beta}^{m_{1}}, \theta_{\beta}^{m_{2}}, \ldots \theta_{\beta}^{m_{t}}$ are all the (maximal) uninterrupted sequences $\theta_{\beta}^{m_{i}} \in \boldsymbol{\theta}$ of values $\beta$ ordered so that the indices are decreasing $m_{1} \geq m_{2} \geq \cdots \geq m_{t}$, then there exists an $i$ : $1 \leq i \leq t$ so that $\tilde{\chi}(x)=1$, if $x \in \theta_{\beta}^{m_{j}}, j \leq i$, and $\tilde{\chi}(x)=0$, if $x \in \theta_{\beta}^{m_{j}}, j>i$ with the exception of at most one $j \geq i$.

By construction as $n \rightarrow \infty$, the process $\left(s_{i}, I_{i}\right), i=1, \ldots, n, \ldots$ is stationary. Since all $\theta_{\alpha}^{m}, \theta_{\beta}^{m}$ are geometrically distributed independent random variables [11], it means explicitly that the probability of a string $\theta_{\beta}^{m}$ is given by

$$
\rho_{\infty}\left(\ldots \theta_{\beta}^{m} \ldots\right)=q(1-q)^{m-1} .
$$

Hence if the total proportion of grains that undergoes a stress-free transformation is $q_{\beta}$, we must have, as $n \rightarrow \infty$

$$
q_{\beta}=r q(1-q)^{k}+\sum_{i=k+1} q(1-q)^{i}=r q(1-q)^{k}+(1-q)^{k+1},
$$

where, due to our assumption of equal probability (4.1), $r$ is the probability that $\tilde{\chi}(x)=1$, if $x \in \theta_{\beta}^{k}$. Therefore $k$ is found so that $(1-q)^{k+1} \leq q_{\beta}<(1-q)^{k}$, i.e., (7.9), and $r$ is found from (7.10).

Since $\theta_{\alpha}^{m}, \theta_{\beta}^{m}$ are independent random variables, the limiting process has exponentially decaying short-range correlations. It implies simultaneously two results: long-range correlations are zero, and short-range correlations decay exponentially with $k$. These correlations are not zero and can be computed explicitly.

Suppose $q_{\beta}=1-q$. This case is slightly more technically complicated, but the methods are the same as in the case $q_{\alpha}=0$. The main new issue is that $s_{i}$ may now take three values, and, therefore, we have to account for three possible different interfaces. Direct computations show that we have here three different situations, depending on the relative value of $\alpha$ and $\beta$. If $\sin ^{2} \alpha>2 \sin ^{2} \beta$, then the maximizer of (7.7) is such that $\tilde{\chi}_{\alpha}(x)=1$ if $x$ belongs to the longest (maximal) uninterrupted strings $\theta_{\alpha}^{m}$. If, however, $\sin ^{2} \alpha<2 \sin ^{2} \beta$, then $\tilde{\chi}_{\alpha}(x)=1$ if $x$ belongs to to the shortest uninterrupted strings $\theta_{\alpha}^{m}$. If $\sin ^{2} \alpha=2 \sin ^{2} \beta$, then there is no difference, and the only statement that is possible to make here is that $\tilde{\chi}_{\alpha}(x)=\tilde{\chi}_{\alpha}(y)$, if $x$ and $y$ belong to the same uninterrupted string $\theta_{\alpha}^{m}$. Due to our assumption of equal probability (4.1), it is possible to conclude that if $\sin ^{2} \alpha=2 \sin ^{2} \beta$, then there is no correlation at all, therefore we will omit the discussion of this case. Combining these arguments with the arguments in the proof of Theorem 7.4 we have the following result.

Theorem 7.5. Consider the Bernoulli trials (4.3). Denote by $\tilde{\chi}_{\alpha}$ and $\tilde{\chi}_{\beta}$ the characteristic function of the grains with $\theta=\alpha$ and $\theta=\beta$, respectively, for which $I=1$. Suppose $U$ is such that the minimizer of the first term in (7.1) satisfies $q_{\beta}=1-q$. Then in the limit $n \rightarrow \infty$ the sequence $\left(s_{i}, I_{i}\right), i=1,2, \ldots, n, \ldots$ is a stationary process with exponentially decaying short-range correlations; long-range correlations are zero, and $\tilde{\chi}_{\beta} \equiv 1$. Moreover, almost surely as $n \rightarrow \infty$, the minimizer of the nearest neighbor model (7.1) satisfies: If $\sin ^{2} \alpha>2 \sin ^{2} \beta$, then

$$
\tilde{\chi}_{\alpha}(x)=\left\{\begin{array}{l}
1, \text { if } x \in \theta_{\alpha}^{m}, m>k, \\
0, \text { if } x \in \theta_{\alpha}^{m}, m<k, \\
1, \text { with probability } r, \text { if } x \in \theta_{\alpha}^{k}, \\
0, \text { with probability } 1-r, \text { if } x \in \theta_{\alpha}^{k},
\end{array}\right.
$$

Copyright (c) by SIAM. Unauthorized reproduction of this article is prohibited. 
where $k=\max (m)$ such that $q_{\alpha}<q^{m}$ and $r$ solves $q_{\alpha}=r(1-q) q^{k}-q^{k+1}$; if $\sin ^{2} \alpha<2 \sin ^{2} \beta$, then

$$
\tilde{\chi}_{\alpha}(x)=\left\{\begin{array}{l}
1, \text { if } x \in \theta_{\alpha}^{m}, m<k, \\
0, \text { if } x \in \theta_{\alpha}^{m}, m>k, \\
1, \text { with probability } r, \text { if } x \in \theta_{\alpha}^{k}, \\
0, \text { with probability } 1-r, \text { if } x \in \theta_{\alpha}^{k},
\end{array}\right.
$$

where $k=\max (m)$ such that $q-q_{\alpha}<q^{m}$ and $r$ solves $q-q_{\alpha}=r(1-q) q^{k}-q^{k+1}$.

\section{Appendix A. Proofs for section 3.}

A.1. Proof of formula (3.4). To obtain the representation (3.4) we begin by decomposing $\boldsymbol{u}=\left(u_{1}, u_{2}\right)$ of the boundary value problem (2.11), (2.13), and (2.14) in the form $\boldsymbol{u}=\tilde{\boldsymbol{u}}+\overline{\boldsymbol{u}}$, where $\tilde{\boldsymbol{u}}$ solves (3.6). The constants $a_{i}, c_{i}, d, f_{i}$, and $g_{i}$ are chosen to satisfy the continuity of the displacement $\tilde{\boldsymbol{u}}$ and traction (condition (2.12)) and, denoting

$$
\tilde{\sigma}_{i j}=c_{i j k l}\left(\tilde{\varepsilon}_{k l}-\varepsilon_{k l}^{\mathrm{T}}\right), \quad \tilde{\varepsilon}_{11}=\partial_{1} \tilde{u}_{1}, \quad \tilde{\varepsilon}_{12}=\tilde{\varepsilon}_{21}=\left(\partial_{2} \tilde{u}_{1}+\partial_{1} \tilde{u}_{2}\right) / 2, \quad \tilde{\varepsilon}_{22}=\partial_{2} \tilde{u}_{2},
$$

the boundary conditions

$$
\tilde{u}_{1}(0, y)=0, \quad \tilde{u}_{1}(1, y)=U, \quad \tilde{u}_{2}(0,0)=0, \quad \tilde{\sigma}_{12}(x, y)=0, \text { for } x=0,1 .
$$

The stresses are

$$
\begin{aligned}
& \tilde{\sigma}_{i, 11}=(\lambda+2 \mathrm{G})\left(a_{i}-\varepsilon_{i, 11}^{\mathrm{T}}\right)+\lambda\left(d-\varepsilon_{i, 22}^{\mathrm{T}}\right), \\
& \tilde{\sigma}_{i, 22}=(\lambda+2 \mathrm{G})\left(d-\varepsilon_{i, 22}^{\mathrm{T}}\right)+\lambda\left(a_{i}-\varepsilon_{i, 11}^{\mathrm{T}}\right), \\
& \tilde{\sigma}_{i, 12}=\mathrm{G}\left(c_{i}-2 \varepsilon_{i, 12}^{\mathrm{T}}\right) .
\end{aligned}
$$

Hence $\left[\tilde{\sigma}_{12}\right]=0$, if $c_{i}=2 \varepsilon_{i, 12}^{\mathrm{T}}$. Similarly $\left[\tilde{\sigma}_{i, 11}\right]=0$, if

$$
a_{i}=\varepsilon_{i, 11}^{\mathrm{T}}+\frac{\lambda}{\lambda+2 \mathrm{G}} \varepsilon_{i, 22}^{\mathrm{T}}+\text { Const }
$$

where the constant can be found from the condition that the displacement of the right boundary is $U$ :

$$
\text { Const }=U-\left\langle\varepsilon_{11}^{\mathrm{T}}\right\rangle-\frac{\lambda}{\lambda+2 \mathrm{G}}\left\langle\varepsilon_{22}^{\mathrm{T}}\right\rangle
$$

Finally,

$$
\begin{gathered}
\left\langle\tilde{\sigma}_{22}\right\rangle=\left\langle(\lambda+2 \mathrm{G})\left(d-\varepsilon_{22}^{\mathrm{T}}\right)+\lambda\left(\frac{\lambda}{\lambda+2 \mathrm{G}} \varepsilon_{22}^{\mathrm{T}}+U-\left\langle\varepsilon_{11}^{\mathrm{T}}\right\rangle-\frac{\lambda}{\lambda+2 \mathrm{G}}\left\langle\varepsilon_{22}^{\mathrm{T}}\right\rangle\right)\right\rangle \\
=(\lambda+2 \mathrm{G})\left(d-\left\langle\varepsilon_{22}^{\mathrm{T}}\right\rangle\right)+\lambda\left(U-\left\langle\varepsilon_{11}^{\mathrm{T}}\right\rangle\right) .
\end{gathered}
$$

Setting $\left\langle\tilde{\sigma}_{22}\right\rangle=0$ we have

$$
d=\left\langle\varepsilon_{22}^{\mathrm{T}}\right\rangle-\frac{\lambda}{\lambda+2 \mathrm{G}}\left(U-\left\langle\varepsilon_{11}^{\mathrm{T}}\right\rangle\right) .
$$

The values of $f_{i}$ and $g_{i}$ in (3.6) are unimportant for our analysis, and we omit them. We have

$$
\tilde{\sigma}_{11}=E_{c}\left(U-\left\langle\varepsilon_{11}^{\mathrm{T}}\right\rangle\right), \tilde{\sigma}_{i, 22}=E_{c}\left(\left\langle\varepsilon_{22}^{\mathrm{T}}\right\rangle-\varepsilon_{i, 22}^{\mathrm{T}}\right), \tilde{\sigma}_{12}=0
$$

Copyright $@$ by SIAM. Unauthorized reproduction of this article is prohibited. 
where the Young's modulus $E_{c}$ is given by (2.16). The elastic misfit energy associated with $\tilde{\boldsymbol{u}}$ is

$$
\frac{1}{E_{c}} \frac{1}{4 L} \int_{-L}^{L} \int_{-1}^{1}\left[\frac{1}{E_{c}}\left(\left(\tilde{\sigma}_{11}\right)^{2}+\left(\tilde{\sigma}_{22}\right)^{2}\right)-2 \frac{\lambda}{4 \mathrm{G}(\lambda+\mathrm{G})} \tilde{\sigma}_{11} \tilde{\sigma}_{22}+\frac{1}{2 \mathrm{G}}\left(\tilde{\sigma}_{12}\right)^{2}\right] d x d y .
$$

Since $\tilde{\sigma}_{12} \equiv 0, \tilde{\sigma}_{11}=$ const, and $\tilde{\sigma}_{22}$ is mean-zero, the above equation becomes

$$
\frac{1}{E_{c}^{2}} \frac{1}{4 L} \int_{-L}^{L} \int_{-1}^{1}\left(\left(\tilde{\sigma}_{11}\right)^{2}+\left(\tilde{\sigma}_{22}\right)^{2}\right) d x d y=\left\langle\left(U-f+\varepsilon_{22}^{\mathrm{T}}\right)^{2}\right\rangle=\left\langle(U-f+s)^{2}\right\rangle,
$$

which is the first term in (3.4).

Let us now find $\overline{\boldsymbol{u}}$. It solves (3.7) on a bounded domain $\Pi_{L}$. A useful periodic setting for (3.7) is obtained by assuming this equation is posed on an infinite strip ]$-\infty, \infty[\times[-L, L]$ with data that is even and periodic in $x$ :

$$
\begin{aligned}
& \text { (a) } \theta(-x)=\theta(x), \theta(x+2)=\theta(x), \\
& \text { (b) } \varepsilon^{\mathrm{T}}(x, y)=\varepsilon^{\mathrm{T}}(-x, y), \varepsilon^{\mathrm{T}}(x+2, y)=\varepsilon^{\mathrm{T}}(x, y) .
\end{aligned}
$$

Thus, $\overline{\boldsymbol{u}}$ equals to the restriction of the solution of (3.7) on an infinite strip $x \in$ ]$-\infty, \infty\left[, y \in[-L, L]\right.$ with periodicity conditions defined by (A.2). Since $\bar{\sigma}_{22}(x, \pm L)$ is a periodic, mean-zero, even function, it can be represented as a cosine Fourier series. The solution on the infinite strip with a sinusoidal symmetric stress $\cos (k \pi x)$ at $y= \pm L$ can be computed explicitly for any $k$ by the Airy function method. Namely, since we are given that $\bar{\sigma}_{22}(x, \pm L)$ is a periodic, mean-zero, even function, it can be represented as

$$
\bar{\sigma}_{22}(x, \pm L)=E_{c} \sum_{k=1}^{\infty} c_{k} \cos (k \pi x), E_{c}=\frac{4 \mathrm{G}(\lambda+\mathrm{G})}{\lambda+2 \mathrm{G}},
$$

where $c_{k}$ are the corresponding Fourier coefficients of $\varepsilon^{\mathrm{T}}(x)$. The solution for the infinite strip with a sinusoidal symmetric stress $\cos (k \pi x)$ at $y= \pm L$ is given (see [19]) by the Airy function

$$
\begin{aligned}
& \Phi^{k}(x, y) \\
& \quad=2 \frac{\cos (k \pi x)}{(k \pi)^{2}} \frac{k \pi y \sinh (k \pi L) \sinh (k \pi y)-[k \pi L \cosh (k \pi L)+\sinh (k \pi L)] \cosh (k \pi y)}{2 k \pi L+\sinh (2 k \pi L)} .
\end{aligned}
$$

This Airy function gives rise to the following stresses

$$
\begin{aligned}
\bar{\sigma}_{11}^{k} & =\frac{\partial^{2} \Phi^{k}}{\partial y^{2}}=d_{11}^{k} \cos (k \pi x), \bar{\sigma}_{12}^{k}=-\frac{\partial^{2} \Phi^{k}}{\partial x \partial y} \\
& =d_{12}^{k} \sin (k \pi x), \bar{\sigma}_{22}^{k}=\frac{\partial^{2} \Phi^{k}}{\partial x^{2}}=d_{22}^{k} \cos (k \pi x),
\end{aligned}
$$

where

$$
\begin{aligned}
d_{11}^{k} & =2 \frac{k \pi y \sinh (k \pi L) \sinh (k \pi y)-[k \pi L \cosh (k \pi L)-\sinh (k \pi L)] \cosh (k \pi y)}{2 k \pi L+\sinh (2 k \pi L)}, \\
\text { (A.3) } d_{12}^{k} & =2 \frac{k \pi y \sinh (k \pi L) \cosh (k \pi y)-k \pi L \cosh (k \pi L) \sinh (k \pi y)}{2 k \pi L+\sinh (2 k \pi L)}, \\
d_{22}^{k} & =2 \frac{-k \pi y \sinh (k \pi L) \sinh (k \pi y)+[k \pi L \cosh (k \pi L)+\sinh (k \pi L)] \cosh (k \pi y)}{2 k \pi L+\sinh (2 k \pi L)} .
\end{aligned}
$$

Copyright $@$ ㅇ by SIAM. Unauthorized reproduction of this article is prohibited. 
Therefore the total stresses are

$$
\begin{gathered}
\sigma_{11}=E_{c}\left(U-\left\langle\varepsilon_{11}^{\mathrm{T}}\right\rangle\right)+E_{c} \sum_{k=1}^{\infty} c_{k} d_{11}^{k} \cos (k \pi x), \\
\sigma_{12}=E_{c} \sum_{k=1}^{\infty} c_{k} d_{12}^{k} \sin (k \pi x), \sigma_{22}=E_{c} \sum_{k=1}^{\infty} c_{k}\left(d_{22}^{k}-1\right) \cos (k \pi x),
\end{gathered}
$$

where in the last equation we have $d_{22}^{k}-1$ instead of $d_{22}^{k}$, because (see (A.1))

$$
\tilde{\sigma}_{22}=-E_{c} \sum_{k=1}^{\infty} c_{k} \cos (k \pi x)
$$

By definition

$$
W=\frac{1}{E_{c}} \frac{1}{4 L} \int_{-L}^{L} \int_{-1}^{1}\left[\frac{1}{E_{c}}\left(\left(\sigma_{11}\right)^{2}+\left(\sigma_{22}\right)^{2}\right)-2 \frac{\lambda}{4 \mathrm{G}(\lambda+\mathrm{G})} \sigma_{11} \sigma_{22}+\frac{1}{2 \mathrm{G}}\left(\sigma_{12}\right)^{2}\right] d x d y .
$$

Since $\int_{-1}^{1} \cos (k \pi x) \cos (m \pi x) d x=\delta_{k m}$

$$
W\left(U, \varepsilon^{\mathrm{T}}\right)=\left\langle\left(U-f+\varepsilon_{22}^{\mathrm{T}}\right)^{2}\right\rangle+\sum_{m=1}^{\infty} c_{m}^{2} \hat{K}_{L}(m),
$$

where

$$
\hat{K}_{L}(m)=\frac{1}{4 L} \int_{-L}^{L}\left[\left(d_{11}^{m}\right)^{2}+d_{22}^{m}\left(d_{22}^{m}-2\right)-\frac{2 \lambda d_{11}^{m}\left(d_{22}^{m}-1\right)}{\lambda+2 \mathrm{G}}+\frac{2(\lambda+\mathrm{G})\left(d_{12}^{m}\right)^{2}}{\lambda+2 \mathrm{G}}\right] d y .
$$

Denoting $a=2 \pi m L$ and using Mathematica ${ }^{\circledR}$ we obtain an explicit form of (A.4):

$$
\begin{gathered}
\hat{K}_{L}(m)=\frac{5 \lambda+9 \mathrm{G}}{2(\lambda+2 \mathrm{G})} S_{1}(a)-\frac{\lambda+\mathrm{G}}{\lambda+2 \mathrm{G}} S_{2}(a), a=2 \pi m L, \text { where } \\
S_{1}(a)=\frac{(\cosh (a)-1)}{a(a+\sinh (a))}, \quad S_{2}(a)=\frac{a^{2}(2+\cosh (a))}{6(a+\sinh (a))^{2}} .
\end{gathered}
$$

A.2. Proof of Lemma 3.1. Using (A.5) and (A.6) from Appendix A.1, direct computations show that the Fourier coefficients of $K_{L}(x)$ (3.5) are given by

$$
\hat{K}_{L}(m)=\frac{B}{L} \frac{1}{m}+O(\exp (-L))
$$

so that, defining

$$
K_{\infty}(x)=\sum_{m=1}^{\infty} \frac{1}{m} \cos (\pi m x)
$$

Copyright (c) by SIAM. Unauthorized reproduction of this article is prohibited. 
(3.8) is satisfied. A closed form expression for this sum is known: $K_{\infty}(x)=$ $-\ln |\sin (\pi x / 2)|$ (see, e.g., [10]), and the Lemma follows.

A.3. Proof of Lemma 3.2. Set $L=l_{0} /(2 n)$, then for $a$ defined in (A.5) we obtain $a=2 \pi m L=l_{0} \pi m / n$. Substitute (A.6) into (A.5) and observe that the Fourier coefficients $\hat{K}_{L}(m)$ depend on the variable $a=l_{0} \pi m / n$. Thus we can introduce the notation

$$
\hat{K}(a):=\hat{K}_{L}(m), a=l_{0} \pi \frac{m}{n} .
$$

In other words the Fourier series of $K_{L}(x)$ can be written in the form

$$
K_{L}(x)=\sum_{m=-\infty}^{\infty} \hat{K}\left(l_{0} \pi \frac{m}{n}\right) \cos (\pi m x)
$$

For $y=n x, y \in[-n, n]$ let

$$
K^{(n)}(y):=\frac{1}{n} K_{L}(y / n)=\frac{1}{n} \sum_{m=-\infty}^{\infty} \hat{K}\left(l_{0} \pi \frac{m}{n}\right) \cos \left(\pi \frac{m}{n} y\right),
$$

and $K^{(n)}(y)=0$ for $|y| \geq n$. Set

$$
\mathcal{K}_{l_{0}}(y)=\frac{1}{2 \pi} \int_{-\infty}^{\infty} \hat{K}\left(l_{0} \zeta\right) \cos (\zeta y) d \zeta, y \in \mathbb{R} .
$$

Note that

$$
\hat{K}\left(l_{0} \zeta\right)=\int_{-\infty}^{\infty} \mathcal{K}_{l_{0}}(y) \cos (\zeta y) d y
$$

provided $\mathcal{K}_{l_{0}}(y)$ is smooth and it decays sufficiently fast as $y \rightarrow \infty$. The function $\mathcal{K}_{l_{0}}(y)$ is exactly the limiting function mentioned in Lemma 3.2; that is, $K^{(n)}(y)=$ $K_{L}(y / n) / n$ converges to $\mathcal{K}_{l_{0}}(y)$ as $n \rightarrow \infty$. Let us first verify properties of $\mathcal{K}_{l_{0}}$ described in Lemma 3.2 and then establish convergence. Direct calculations using (A.5) show that $\hat{K}(a)>0$ for all physical choices of the Lamé constants, so that, by Bochner's theorem [18, Vol. 1], $\mathcal{K}_{l_{0}}$ is positive-definite. We verify (3.10) by applying the Paley-Wiener type Theorems [18, Vol. 1]. Indeed, from (A.6) it follows that the Fourier transform of $\mathcal{K}_{l_{0}}$ can be analytically extended into a finite strip $|\operatorname{Im}(a)| \leq c_{2}$ around the real axis provided there is no solution of the equation $a+\sinh (a)=0$, $a \neq 0$, or, equivalently

$$
\operatorname{Re}(a)=-\sinh (\operatorname{Re}(a)) \cos (\operatorname{Im}(a)), \operatorname{Im}(a)=-\cosh (\operatorname{Re}(a)) \sin (\operatorname{Im}(a)), a \neq 0 .
$$

There is no solution of the last equation in (A.9) at least in the strip $|\operatorname{Im}(a)| \leq \pi$. This implies the exponential decay (3.10) of $\mathcal{K}_{l_{0}}(y)$ by Paley-Wiener theorems. Finally, for every $n$ consider the $2 n$-periodization of $\mathcal{K}_{l_{0}}(y)$ :

$$
\mathcal{K}_{l_{0}}^{(n)}(y)=\sum_{k=-\infty}^{+\infty} \mathcal{K}_{l_{0}}(y+2 n k) .
$$

Copyright (C) by SIAM. Unauthorized reproduction of this article is prohibited. 
Since $\mathcal{K}_{l_{0}}(y)$ decays exponentially as $y \rightarrow \infty$, we have that $\mathcal{K}_{l_{0}}^{(n)}(y)$ is a smooth $2 n$ periodic function and its Fourier coefficients

$$
\begin{aligned}
& \hat{\mathcal{K}}_{l_{0}}^{(n)}(m)=\frac{1}{n} \int_{-n}^{n} \mathcal{K}_{l_{0}}^{(n)}(y) \cos \left(\pi \frac{m}{n} y\right) d y \\
&=\frac{1}{n} \int_{-n}^{n}\left(\sum_{k=-\infty}^{+\infty} \mathcal{K}_{l_{0}}(y+2 n k)\right) \cos \left(\pi \frac{m}{n} y\right) d y \\
&=\frac{1}{n} \int_{-\infty}^{\infty} \mathcal{K}_{l_{0}}(y) \cos \left(\pi \frac{m}{n} y\right) d y=\frac{1}{n} \hat{K}\left(l_{0} \pi \frac{m}{n}\right)=\frac{1}{n} \hat{K}_{L}(m) .
\end{aligned}
$$

Thus $\mathcal{K}_{l_{0}}^{(n)}(y)=K_{L}(y / n) / n=K^{(n)}(y)$ on $[-n, n]$. Therefore

$$
\begin{aligned}
\left\|K_{L}(x)-n \mathcal{K}_{l_{0}}(n x)\right\|_{L_{\infty}([-1,1])} & =n\left\|K^{(n)}(y)-\mathcal{K}_{l_{0}}(y)\right\|_{L_{\infty}([-n, n])} \\
& =n\left\|\mathcal{K}_{l_{0}}^{(n)}(y)-\mathcal{K}_{l_{0}}(y)\right\|_{L_{\infty}([-n, n])} \\
& =n\left\|\sum_{k \neq 0} \mathcal{K}_{l_{0}}(y+2 n k)\right\|_{L_{\infty}([-n, n])} \leq C n \sum_{k=1}^{\infty} e^{-c k n} \\
& \leq C n e^{-c n} \rightarrow 0,
\end{aligned}
$$

as $n \rightarrow \infty$, and $C=C\left(l_{0}\right)>0$ is independent of $n$.

A.4. Proof of Proposition 3.3. For any $U$ and $\varepsilon^{T}$ we can estimate the error of the truncation (3.14) as

$$
\begin{gathered}
\left|W_{n}\left(U, \varepsilon^{\mathrm{T}}\right)-W_{n}^{k_{0}}\left(U, \varepsilon^{\mathrm{T}}\right)\right| \leq c \sum_{k>k_{0}}\left|\lambda_{k}(n)\right|\left\langle(s-\langle s\rangle)^{2}\right\rangle \\
\leq c \exp \left(-k_{0}\right)\left\langle(s-\langle s\rangle)^{2}\right\rangle \leq c \exp \left(-k_{0}\right) .
\end{gathered}
$$

Since

$$
W_{n}^{k_{0}}\left(U, \varepsilon_{k_{0}}^{\mathrm{T}}\right)=\min _{\tilde{\varepsilon}^{\mathrm{T}} \in \tilde{\Omega}_{n}(\theta)} W_{n}^{k_{0}}\left(U, \tilde{\varepsilon}^{\mathrm{T}}\right)
$$

we have

$$
W_{n}\left(U, \varepsilon^{\mathrm{T}}\right) \leq \min _{\tilde{\boldsymbol{\varepsilon}}^{\mathrm{T}} \in \tilde{\Omega}_{n}(\theta)} W_{n}\left(U, \tilde{\boldsymbol{\varepsilon}}^{\mathrm{T}}\right)+c \exp \left(-k_{0}\right)=W_{n}(U, \boldsymbol{\theta})+c \exp \left(-k_{0}\right) .
$$

By definition of the minimizer $W_{n}(U, \boldsymbol{\theta}) \leq W_{n}\left(U, \varepsilon^{\mathrm{T}}\right)$. This implies (3.15).

\section{Appendix B. Proofs for section 5.}

B.1. Verification of (5.11). For a given product measure $\tilde{\mu}$ we have

$$
\int I_{1} I_{2} d \tilde{\mu}=\left(\int I_{1} d \tilde{\mu}\right)^{2}, \int s_{1} I_{2} d \tilde{\mu}=\left(\int s_{1} d \tilde{\mu}\right)\left(\int I_{1} d \tilde{\mu}\right),
$$

because the events in the first and the second grains are independent and identically distributed. Hence

$$
\begin{aligned}
\int I_{1} I_{2} d \tilde{\mu} & =\left(\int_{0}^{\pi / 4} \chi(\theta) d \rho(\theta)\right)^{2} \\
& =f^{2}, \quad \int\left(U+s_{1}\right) I_{2} d \tilde{\mu}=f\left(\int_{0}^{\pi / 4}(U+s(\theta)) \chi(\theta) d \rho(\theta)+(1-f) U\right) .
\end{aligned}
$$

Copyright $@$ ㅇ by SIAM. Unauthorized reproduction of this article is prohibited. 
It gives

$$
\begin{aligned}
\int F( & \left(U, s_{1}, I_{1}, I_{2}\right) d \tilde{\mu} \\
= & \int\left(\left(U+s_{1}\right)^{2}-2\left(U+s_{1}\right) I_{2}+I_{1} I_{2}\right) d \tilde{\mu} \\
= & \int_{0}^{\pi / 4}(U+s(\theta))^{2} \chi(\theta) d \rho(\theta)+(1-f) U^{2} \\
& \quad-2 f\left(\int_{0}^{\pi / 4}(U+s(\theta)) \chi(\theta) d \rho(\theta)+(1-f) U\right)+f^{2} \\
= & \int_{0}^{\pi / 4}\left((U+s(\theta)-f)^{2}-f^{2}\right) \chi(\theta) d \rho(\theta)+(1-f)\left(U^{2}-2 U f\right)+f^{2} \\
= & \int_{0}^{\pi / 4}(U+s(\theta)-f)^{2} \chi(\theta) d \rho(\theta)+(U-f)^{2}(1-f),
\end{aligned}
$$

where the last equality is obtained by noting the following identity:

$$
f^{2}=f^{2}(1-f)+\int_{0}^{\pi / 4} f^{2} \chi(\theta) d \rho(\theta)
$$

B.2. Proof of Lemma 5.3. The proof is the direct evaluation and comparison of all possible scenarios in (5.12). Using (5.12), we simply consider four functions of $U, q, q_{\alpha}$, and $q_{\beta}$ :

$$
\begin{gathered}
W_{1}=\left(U-q_{\alpha}-q_{\beta}+\cos ^{2} \alpha\right)^{2} q_{\alpha} \\
+\left(U-q_{\alpha}-q_{\beta}+\cos ^{2} \beta\right)^{2} q_{\beta}+\left(U-q_{\alpha}-q_{\beta}\right)^{2}\left(1-q_{\alpha}-q_{\beta}\right), \\
W_{2}=\left(U-q_{\alpha}-q_{\beta}+\cos ^{2} \alpha\right)^{2} q_{\alpha} \\
+\left(U-q_{\alpha}-q_{\beta}+\sin ^{2} \beta\right)^{2} q_{\beta}+\left(U-q_{\alpha}-q_{\beta}\right)^{2}\left(1-q_{\alpha}-q_{\beta}\right), \\
+\left(U-q_{\alpha}-q_{\beta}+\cos ^{2} \beta\right)^{2} q_{\beta}+\left(U-q_{\alpha}+q_{\beta}\right)^{2}\left(1-q_{\alpha}-q_{\beta}\right), \\
W_{3}=\left(U-q_{\alpha}-q_{\beta}+\sin ^{2} \alpha\right)^{2} q_{\alpha} \\
+\left(U-q_{\alpha}-q_{\beta}+\sin ^{2} \alpha\right)^{2} q_{\alpha} \\
\left.+q_{\beta}+\sin ^{2} \beta\right)^{2} q_{\beta}+\left(U-q_{\alpha}-q_{\beta}\right)^{2}\left(1-q_{\alpha}-q_{\beta}\right),
\end{gathered}
$$

and compare their values for each fixed $U$ and $q$, where $q_{\alpha}$ and $q_{\beta}$ are in the range (5.13). It is easy to check that the minimum is always achieved for $W_{4}$; hence equation (5.14) is satisfied.

Copyright $@$ ㅇ by SIAM. Unauthorized reproduction of this article is prohibited. 


\section{Appendix C. Proofs for section 6.}

C.1. Proof of Lemma 6.4. Since the Fourier coefficients of a convolution equal the products of the Fourier coefficients, and since from (A.8) we know the Fourier coefficients of $K_{\infty}$ are $\leq 1$ in absolute value, and in view of Plancherel's theorem, we have

$$
\int_{-1}^{1} \int_{-1}^{1}(s(x)-\langle s\rangle)(s(t)-\langle s\rangle) K_{\infty}(x-t) d x d t \leq \int_{-1}^{1}(s(x)-\langle s\rangle)^{2} d x,
$$

and $|s(x)| \leq 1$, for any $\delta>0$, there exists $L_{0}>0$, so that for any $L \geq L_{0}$

$$
\left|\frac{B}{L} \bar{W}_{n}\left(U, \varepsilon^{\mathrm{T}}\right)\right| \leq \delta
$$

Hence for any $s=\varepsilon_{22}^{\mathrm{T}}$

$$
\left\langle(U-f+s)^{2}\right\rangle<W_{n}\left(U, \varepsilon^{\mathrm{T}}\right) \leq\left\langle(U-f+s)^{2}\right\rangle+\delta .
$$

It follows that

$$
\min _{s, f}\left\langle(U-f+s)^{2}\right\rangle<\min _{s, f} W_{n}\left(U, \varepsilon^{\mathrm{T}}\right) \leq \min _{s, f}\left\langle(U-f+s)^{2}\right\rangle+\delta
$$

for any sequence of angles $\boldsymbol{\theta}$. Applying Lemma 5.5 we complete the proof of (6.5). Equality (6.4) follows from direct computations as in Lemma 5.3.

C.2. Proof of Lemma 6.6. Suppose (6.18) and (6.19) do not hold. It means that there exists $\delta>0$ such that for every $\delta^{\prime}>0$ there is a sequence of sets $A_{n_{k}} \in \sigma_{n_{k}}^{\theta}$, $\left\{n_{k}\right\} \rightarrow \infty$ with probability $\rho_{n_{k}}\left(A_{n_{k}}\right)>2 C>0$ such that for every fixed $\boldsymbol{\theta} \in A_{n_{k}}$ (or, equivalently, $\left.\left(\chi_{\alpha}, \chi_{\beta}\right) \in A_{n_{k}}\right)$ there is a (at least one) maximizer $\tilde{\chi}_{\alpha}, \tilde{\chi}_{\beta}$ of (6.17) such that

$$
\min \left(e_{1}, e_{2}\right)>\delta
$$

where

$e_{1}=\int_{0}^{1}\left(\left|\tilde{\chi}_{\alpha}-\chi_{q_{\alpha}}^{1}\right| \chi_{\alpha}+\left|\tilde{\chi}_{\beta}-\chi_{q_{\beta}}^{1}\right| \chi_{\beta}\right) d x, e_{2}=\int_{0}^{1}\left(\left|\tilde{\chi}_{\alpha}-\chi_{q_{\alpha}}^{2}\right| \chi_{\alpha}+\left|\tilde{\chi}_{\beta}-\chi_{q_{\beta}}^{2}\right| \chi_{\beta}\right) d x$

$\chi_{q_{\alpha}}^{i}$ and $\chi_{q_{\beta}}^{i}$ are defined in (6.14) and (6.15), respectively. Since $K(x) \in L^{1}[-1,1]$ is fixed and $\tilde{\chi}_{\alpha} \chi_{\alpha}, \tilde{\chi}_{\beta} \chi_{\beta}$ are uniformly bounded in $L^{\infty}[-1,1]$, for any $\delta_{1}>0$ there exists $h>0$ so that

$$
\begin{aligned}
& \left|\int_{-1}^{1} \int_{-1}^{1} \varepsilon_{\alpha}(x) \varepsilon_{\alpha}(t) K(x-y) d x d t-\int_{-1}^{1} \int_{-1}^{1} \bar{\varepsilon}_{\alpha}(x) \bar{\varepsilon}_{\alpha}(t) K(x-y)\right| d x d t \mid<\delta_{1} \\
& \left|\int_{-1}^{1} \int_{-1}^{1} \varepsilon_{\alpha}(x) \varepsilon_{\beta}(t) K(x-y)\right| d x d t-\int_{-1}^{1} \int_{-1}^{1} \bar{\varepsilon}_{\alpha}(x) \bar{\varepsilon}_{\beta}(t) K(x-y)|d x d t|<\delta_{1} \\
& \left|\int_{-1}^{1} \int_{-1}^{1} \varepsilon_{\beta}(x) \varepsilon_{\beta}(t) K(x-y)\right| d x d t-\int_{-1}^{1} \int_{-1}^{1} \bar{\varepsilon}_{\beta}(x) \bar{\varepsilon}_{\beta}(t) K(x-y)|d x d t|<\delta_{1}
\end{aligned}
$$

where

$$
\bar{\varepsilon}_{\alpha}(x)=\frac{1}{2 h} \int_{-h}^{h} \bar{\varepsilon}_{\alpha}(x+t) d t, \bar{\varepsilon}_{\beta}(x)=\frac{1}{2 h} \int_{-h}^{h} \bar{\varepsilon}_{\beta}(x+t) d t .
$$

Copyright (c) by SIAM. Unauthorized reproduction of this article is prohibited. 
By the law of the large numbers, for any $\delta_{2}>0$ there is $\bar{A}_{n_{k}} \in A_{n_{k}}, \rho_{n_{k}}\left(A_{n_{k}}\right)>C$ so that for every $\boldsymbol{\theta} \in \bar{A}_{n_{k}}$ (or, equivalently, $\left(\chi_{\alpha}^{n_{k}}, \chi_{\beta}^{n_{k}}\right) \in \bar{A}_{n_{k}}$ ) and $n_{k}>N_{0}$, we have

$$
\bar{\varepsilon}_{\alpha}(x)=q \chi_{\alpha}(x, \boldsymbol{\theta})-q_{\alpha}(\boldsymbol{\theta}), \bar{\varepsilon}_{\beta}(x)=(1-q) \chi_{\beta}(x, \boldsymbol{\theta})-q_{\alpha}(\boldsymbol{\theta}),
$$

where (random in $\boldsymbol{\theta} \in \bar{A}_{n_{k}}$ ) functions $\chi_{\alpha}(x, \boldsymbol{\theta}), \chi_{\beta}(x, \boldsymbol{\theta})$ satisfy

$$
0 \leq \chi_{\alpha}(x, \boldsymbol{\theta}) \leq 1+\delta_{2}, 0 \leq \chi_{\beta}(x, \boldsymbol{\theta}) \leq 1+\delta_{2},
$$

and $\min \left(e_{1}, e_{2}\right)>\delta-\delta_{2}$ where

$$
\begin{aligned}
e_{1} & =\int_{0}^{1}\left(\left|\chi_{\alpha}(x, \boldsymbol{\theta})-\chi_{q_{\alpha}}^{1}\right|+\left|\chi_{\beta}(x, \boldsymbol{\theta})-\chi_{q_{\beta}}^{1}\right|\right) d x, e_{2} \\
& =\int_{0}^{1}\left(\left|\chi_{\alpha}(x, \boldsymbol{\theta})-\chi_{q_{\alpha}}^{2}\right|+\left|\chi_{\beta}(x, \boldsymbol{\theta})-\chi_{q_{\beta}}^{2}\right|\right) d x .
\end{aligned}
$$

The classical Riesz rearrangement inequality (6.11) implies that when $\delta_{1}$ and $\delta_{2}$ are sufficiently small, there is $\delta^{\prime}>0$ so that for every $\boldsymbol{\theta} \in \bar{A}_{n_{k}}$

$$
\begin{aligned}
& \int_{-1}^{1} \int_{-1}^{1}\left(a \varepsilon_{\alpha}(x)+b \varepsilon_{\beta}(x)\right)\left(a \varepsilon_{\alpha}(t)+b \varepsilon_{\beta}(t)\right) K(x-t) d x d t \\
& \leq \int_{-1}^{1} \int_{-1}^{1}\left(a \varepsilon_{\alpha}^{1}(x)+b \varepsilon_{\beta}^{1}(x)\right)\left(a \varepsilon_{\alpha}^{1}(t)+b \varepsilon_{\beta}^{1}(t)\right) K(x-t) d x d t-\delta^{\prime},
\end{aligned}
$$

where

$$
\varepsilon_{\alpha}^{1}(x)=\chi_{q_{\alpha}}^{1} \chi_{\alpha}, \varepsilon_{\alpha}^{1}(x)=\chi_{q_{\beta}}^{1} \chi_{\beta} .
$$

Hence we have that $\tilde{\chi}_{\alpha}$ and $\tilde{\chi}_{\beta}$ with (C.1) and (C.2) cannot be maximizers of (6.17). This leads to contradiction with our assumption that (6.18) and (6.19) do not hold.

Acknowledgments. We thank Alexei Borodin and Omri Sarig for useful discussions. We are grateful to anonymous referees for their useful suggestions.

\section{REFERENCES}

[1] A. Baernstein, II, Convolution and rearrangement on the circle, Complex Variables Theory Appl., 12 (1989), pp. 33-37.

[2] K. Bhattacharya, B. Li, And M. Luskin, The simply laminated microstructure in martensitic crystals that undergo a cubic-to-orthorhombic phase transformation, Arch. Ration. Mech. Anal., 149 (1999), pp. 123-154.

[3] K. Bhattacharya and R. V. Kohn, Elastic energy minimization and the recoverable strains of polycrystalline shape-memory materials, Arch. Ration. Mech. Anal., 139 (1997), pp. 99180.

[4] J. S. Bowles and J. K. Mackenzie, The crystallography of martensite transformations I, Acta Metall., 2 (1954), pp. 129-137.

[5] J. S. Bowles AND J. K. MACKenZIE, The crystallography of martensite transformations III. Face-centered cubic to body-centered tetragonal transformations, Acta Metall., 2 (1954), pp. 224-234.

[6] O. P. Bruno and G. H. Goldsztein, A fast algorithm for the simulation of polycrystalline misfits: Martensitic transformations in two space dimensions, R. Soc. Lond. Proc. Ser. A Math. Phys. Eng. Sci., 455 (1999), pp. 4245-4276.

[7] O. P. BRuno And G. H. Goldsztein, Numerical simulation of martensitic transformations in two- and three-dimensional polycrystals, The J. R. Willis 60th anniversary volume, J. Mech. Phys. Solids, 48 (2000), pp. 1175-1201.

Copyright $@$ by SIAM. Unauthorized reproduction of this article is prohibited. 
[8] O. P. Bruno and G. H. Goldsztein, A fast algorithm for the simulation of polycrystalline misfits. II. Martensitic transformations in three space dimensions, R. Soc. Lond. Proc. Ser. A Math. Phys. Eng. Sci., 460 (2004), pp. 1613-1630.

[9] O. P. BRuno, F. Reitich, And P. Leo, The overall elastic energy of polycrystalline martensitic solids, J. Mech. Phys. Solids, 44 (1996), pp. 1051-1101.

[10] B. Demidovich, ED., Problems in Mathematical Analysis, Peace Publishers, Moscow, 1965.

[11] W. Feller, An Introduction to Probability Theory and Its Applications, 3rd ed., Vol. I, John Wiley \& Sons, New York-London-Sydney, 1968.

[12] G. H. Hardy, J. E. Littlewood, And G. Pólya, Inequalities, 2nd ed., Cambridge University Press, Cambridge, 1952.

[13] E. Hewitt and L. J. Savage, Symmetric measures on Cartesian products, Trans. Amer. Math. Soc., 80 (1955), pp. 470-501.

[14] E. H. Lieb And M. Loss, Analysis, 2nd ed., Graduate Studies in Mathematics, 14, American Mathematical Society, Providence, RI, 2001.

[15] J. K. Mackenzie And J. S. Bowles, The crystallography of martensite transformations II, Acta Metall., 2 (1954), pp. 138-147.

[16] G. W. Milton, The Theory of Composites, Cambridge University Press, Cambridge, 2002.

[17] R. L. Patterson and C. M. Wayman, Internal twinning in ferrous martensites, Acta Met., 12 (1964), pp. 1306-11.

[18] M. Reed And B. Simon, Methods of Modern Mathematical Physics, 2nd ed., Academic Press, Inc., New York, 1980.

[19] S. Timoshenko and J. N. Goodier, Theory of Elasticity, 2nd ed., McGraw-Hill Book Company, Inc., New York, Toronto, London, 1951.

[20] V. P. Smyshlyaev and J. R. Willis, A "non-local" variational approach to the elastic energy minimization of martensitic polycrystals, R. Soc. Lond. Proc. Ser. A Math. Phys. Eng. Sci. 454 (1998), pp. 1573-1613.

[21] M. S. Wechsler, D. S. Lieberman, and T. A. Read, On the theory of the formation of martensite, AIME Trans. J. Metals, 197 (1953), pp. 1503-1515.

[22] J. R. Willis, Variational and related methods for the overall properties of composites, Adv. Appl. Mech., 21 (1981), pp. 1-78.

Copyright (c) by SIAM. Unauthorized reproduction of this article is prohibited. 